Supplementary Information

\title{
Color-Tuning and Near-Sunlight White Emission in Highly Stable Rod-Spacer MOFs with Defective Dicubane Based Lead(II)-Carboxyl Chains
}

Zheng Yin,,${ }^{*} \dagger$ Wei-Min Ma,${ }^{\dagger}$ Ce Wang, ${ }^{\dagger}$ Xiao-Peng Luo,${ }^{\dagger}$ Xiao-Ting Hu,${ }^{\dagger}$ Hui-Li Cao, ${ }^{\dagger}$ Xi-Yao Li, ${ }^{\dagger}$

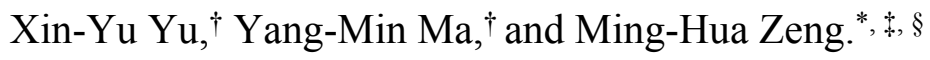

$\dagger$ College of Chemistry and Chemical Engineering, Shaanxi Key Laboratory of Chemical Additives for Industry, Shaanxi University of Science and Technology, Xi'an 710021, P. R. China.

\$ Hubei Collaborative Innovation Center for Advanced Organic Chemical Materials, Ministry of Education Key Laboratory for the Synthesis and Application of Organic Functional Molecules, College of Chemistry and Chemical Engineering, Hubei University, Wuhan, 430062, P. R. China.

$\S$ Key Laboratory for the Chemistry and Molecular Engineering of Medicinal Resources, School of Chemistry and Pharmaceutical Sciences, Guangxi Normal University, Guilin, 541004, P. R. China. 
Table S1 Statistical of typical porous Pb-MOFs and their fluorescent properties in the literatures.

\begin{tabular}{|c|c|c|c|c|}
\hline No & Formula & Structure Features & Fluorescent Properties & Ref. \\
\hline 1 & $\begin{array}{l}{\left[\left(\mathrm{CH}_{3}\right)_{2} \mathrm{NH}_{2}^{+}\right]_{3}\left(\left[\mathrm{~Pb}_{2} \mathrm{Cl}_{3}^{+}\right]\right.} \\
{\left[\mathrm{O}_{2} \mathrm{C}\left(\mathrm{C}_{6} \mathrm{H}_{4}\right) \mathrm{CO}_{2}^{-}\right]_{2}}\end{array}$ & $\begin{array}{l}\text { Rod-spacer framework } \\
\text { with deformable lead } \\
\text { halide chain }\end{array}$ & $\begin{array}{l}\text { Broadband emission in } 400- \\
800 \mathrm{~nm} \text { with FWHM of } 207 \\
\mathrm{~nm} \text { and CIE of }(0.37,0.39) \text {, } \\
\text { under excitation of } 380 \mathrm{~nm} \text {. } \\
\text { Mechanism: self-trapped } \\
\text { excitons from structural } \\
\text { deformation in excited state. }\end{array}$ & [1] \\
\hline 2 & $\begin{array}{l}\mathrm{PbL} 1\left(\mathrm{~L} 1=\mathrm{CH}_{3} \mathrm{SCH}_{2} \mathrm{CH}_{2} \mathrm{~S} \text {-attached }\right. \\
\text { 1,4-benzenedicarboxylic acid) }\end{array}$ & $\begin{array}{l}\text { 3D coordination net } \\
\text { interlinked by } \mathrm{Pb}- \\
\text { carboxyl chain }\end{array}$ & $\begin{array}{l}\text { Broad emission with maxima } \\
\text { situated at } 459 \text { and } 515 \mathrm{~nm} \text {, } \\
\text { with CIE of }(0.27,0.30) \text {, } \\
\text { under excitation of } 300 \mathrm{~nm} \text {. } \\
\text { Mechanism: intraligand } \\
\text { transitions, LMCT and metal- } \\
\text { centered transitions. }\end{array}$ & [2] \\
\hline 3 & $\begin{array}{l}{\left[\mathrm{Pb}(\mu \text {-bpy })\left(\mu-\mathrm{O}_{2} \mathrm{CCF}_{3}\right)_{2}\right] \cdot 0.5 \mathrm{CHCl}_{3}} \\
\text { (bpy }=4,4^{\prime} \text {-bipyridine) }\end{array}$ & $\begin{array}{l}\text { Doubly interpenetrated } \\
\text { framework with tetra- } \\
\text { connected } \\
\mathrm{Pb}_{4}\left(\mathrm{O}_{2} \mathrm{CCF}_{3}\right)_{8} \text { cluster }\end{array}$ & $\begin{array}{l}\text { Broad emission with maxima } \\
\text { situated at } 512 \text { and } 574 \mathrm{~nm} \text {, } \\
\text { with CIE of }(0.33,0.39) \text {, } \\
\text { under excitation of } 300 \mathrm{~nm} \text {. } \\
\text { Mechanism: LMCT and } \\
\text { metal-centered transitions. }\end{array}$ & [3] \\
\hline 4 & $\begin{array}{l}{\left[\mathrm{Pb}_{4}(2,6-\mathrm{ndc})_{3} \mathrm{Cl}_{2}\right]_{\mathrm{n}}\left(2,6-\mathrm{H}_{2} \mathrm{ndc}=2,6-\right.} \\
\text { naphthalene dicarboxylate })\end{array}$ & $\begin{array}{l}\text { 3D pillared-layer } \\
\text { framework }\end{array}$ & $\begin{array}{l}\text { Multipeak emissions } \\
\text { centered at 434, 536, } 578 \text { and } \\
633 \mathrm{~nm} \text {, upon excitation at } \\
408 \mathrm{~nm} \text {. } \\
\text { Mechanism: strong } \pi-\pi \\
\text { interaction of the ligands. } \\
\end{array}$ & [4] \\
\hline 11 & $\begin{array}{l}\left.\left[\mathrm{Pb}_{6} \mathrm{O}_{2}\right) \mathrm{L}_{4}\right] \cdot 2 \mathrm{EtOH}\left(\mathrm{H}_{2} \mathrm{~L}=\text { biphenyl- }\right. \\
\text { 2,2'-dimethoxy-4,4'-dicarboxylic } \\
\text { acid) }\end{array}$ & $\begin{array}{l}{\left[\mathrm{Pb}_{6} \mathrm{O}_{2}\left(\mathrm{CO}_{2}\right)_{4}\right] \text { cluster }} \\
\text { based framework with } \\
\text { void of } 21.7 \%\end{array}$ & $\begin{array}{l}\text { Single emission centered at } \\
534 \mathrm{~nm} \text {, upon excitation at } \\
419 \mathrm{~nm} \text {. } \\
\text { Mechanism: intraligand } \\
\text { transitions. }\end{array}$ & [5] \\
\hline 13 & $\begin{array}{l}{\left[\mathrm{Pb}_{4}(\mathrm{OH})_{4}(\mathrm{BDT})_{2}\right] \cdot 2 \mathrm{H}_{2} \mathrm{O}(\mathrm{BDT}=1,4-} \\
\text { benzeneditetrazol-5-yl })\end{array}$ & $\begin{array}{l}\text { 2-fold interpenetrating } \\
(10,3)-d \text { (or utp) } \\
\text { topology }\end{array}$ & $\begin{array}{l}\text { Single emission centered at } \\
552 \mathrm{~nm} \text {, up excitation at } 364 \\
\mathrm{~nm} . \\
\text { Mechanism: LMCT }\end{array}$ & [6] \\
\hline 14 & $\begin{array}{l}{\left[\mathrm{Pb}\left(\mathrm{BDC}-\mathrm{Cl}_{4}\right)(\mathrm{MeOH})_{2}\right]_{\mathrm{n}} \quad\left(\mathrm{H}_{2} \mathrm{BDC}-\right.} \\
\mathrm{Cl}_{4}=2,3,5,6 \text {-tetrachloro-1,4-benzene- } \\
\text { dicarboxylic acid })\end{array}$ & $\begin{array}{l}\text { Binodal four-connected } \\
\text { pts net }\end{array}$ & $\begin{array}{l}\text { Emissions centered at } 477 \\
\mathrm{~nm} \text {, with weaker shoulder } \\
\text { peak around } 510 \mathrm{~nm} \text {, up } \\
\text { excitation at } 336 \mathrm{~nm} \text {. } \\
\text { Mechanism: ligand-centered } \\
\text { and intraligand transitions. }\end{array}$ & [7] \\
\hline 15 & $\begin{array}{l}{\left[\mathrm{Pb}(\text { pydc })\left(\mathrm{H}_{2} \mathrm{O}\right)\right]\left(\mathrm{H}_{2} \text { pydc }=\text { pyridine- }\right.} \\
\text { 2,5-dicarboxylic acid })\end{array}$ & $\begin{array}{l}3 \mathrm{D} \text { framework with } 1 \mathrm{D} \\
\text { chains }\end{array}$ & $\begin{array}{l}\text { One distinct maximum at } 441 \\
\text { nm with three broad } \\
\text { shoulders around } 470,520 \text {, } \\
\text { and } 563 \mathrm{~nm} \text {, given CIE of } \\
(0.29,0.30) . \\
\text { Mechanism: LMCT and } \\
\text { intraligand transitions. }\end{array}$ & [8] \\
\hline
\end{tabular}




\begin{tabular}{|c|c|c|c|c|}
\hline 2 & {$\left[\mathrm{~Pb}_{6} \mathrm{O}_{2}\left(\mathrm{C}_{69} \mathrm{H}_{48} \mathrm{O}_{12} \mathrm{~S}_{4}\right)_{2}(\mathrm{DMA})_{3}\left(\mathrm{H}_{2} \mathrm{O}\right)_{2}\right]$} & $\begin{array}{l}(4,8) \text {-connected net with } \\
{\left[\mathrm{Pb}_{6} \mathrm{O}_{2}\left(\mathrm{CO}_{2}\right)_{8}\right] \text { cluster }}\end{array}$ & \multirow{7}{*}{$\begin{array}{l}\text { No reported fluorescent } \\
\text { properties. }\end{array}$} & [9] \\
\hline 6 & $\begin{array}{l}{\left[\mathrm{Pb}_{9}(\mathrm{~L})_{3}\left(\mathrm{CH}_{3} \mathrm{COO}\right)_{2}\left(\mathrm{H}_{2} \mathrm{O}\right)_{4}\right] \cdot 2\left(\mathrm{CH}_{3}\right)_{2}} \\
\mathrm{NH}_{2}{ }^{+} \cdot 12 \mathrm{DMA}\left(\mathrm{H}_{6} \mathrm{~L}=\text { Hexakis }(4-\right. \\
\text { ethoxycarbonyl-3-methoxy-phenoxy) } \\
\text { cyclotriphosph azene) }\end{array}$ & $\begin{array}{l}(4,12) \text {-connected } \\
\text { network of } \mathrm{Al}_{3} \mathrm{La} \\
\text { topology }\end{array}$ & & {$[10]$} \\
\hline 7 & $\begin{array}{l}{\left[\mathrm{Pb}_{4} \mathrm{Pd}\left(\mathrm{C}_{21} \mathrm{H}_{14} \mathrm{~N}_{4} \mathrm{O}_{4}\right)_{2} \mathrm{Br}_{4}\left(\mathrm{C}_{2} \mathrm{O}_{4}\right)\right]} \\
\cdot 11 \mathrm{H}_{2} \mathrm{O}\end{array}$ & $(4,8)$-connected net & & [11] \\
\hline 8 & $\begin{array}{l}{[\mathrm{Pb}(\mathrm{HBTI})]_{\mathrm{n}}(\mathrm{HBTI}=4,5 \text {-bis }(1 \mathrm{H}-} \\
\text { tetrazole })-1 \mathrm{H} \text {-imidazole })\end{array}$ & $\begin{array}{l}\text { 2-nodal }(6,6) \text {-connected } \\
\text { net }\end{array}$ & & {$[12]$} \\
\hline 9 & $\begin{array}{l}{\left[\mathrm{Pb}_{2}\left(\mathrm{H}_{2} \mathrm{TCPP}\right)\right] \cdot 4 \mathrm{DMF} \cdot \mathrm{H}_{2} \mathrm{O}\left(\mathrm{H}_{6} \mathrm{TCPP}\right.} \\
=5,10,15,20 \text {-tetra(carboxyphenyl) } \\
\text { porphyrin) }\end{array}$ & $\begin{array}{l}\text { Rod spacer with Pb- } \\
\text { carboxyl chain and void } \\
\text { of } 39.5 \% \text {. }\end{array}$ & & {$[13]$} \\
\hline 10 & $\begin{array}{l}{\left[\mathrm{Pb}(\mathrm{BTO})\left(\mathrm{H}_{2} \mathrm{O}\right)\right]_{\mathrm{n}}} \\
\mathrm{BTO}=5,5^{\prime} \text {-bistetrazole-1,1'-diolate }\end{array}$ & $\begin{array}{l}\text { 3D parallelogram } \\
\text { porous framework }\end{array}$ & & [14] \\
\hline 12 & $\begin{array}{l}{\left[\mathrm{PbL}_{2}\right] \cdot \mathrm{DMF} \cdot 2 \mathrm{H}_{2} \mathrm{O} \quad(\mathrm{HL}=\mathrm{N}-(4-} \\
\text { carboxy-phenyl)isonicotinamide } \\
\text { oxide) } \\
\text { oxide }\end{array}$ & $\begin{array}{l}\text { Rod-spacer with } \\
{\left[\mathrm{PbO}_{2}\right]_{\infty} \text { chain and void }} \\
\text { of } 40.3 \%\end{array}$ & & {$[15]$} \\
\hline
\end{tabular}

\section{Reference:}

[1] Peng, C.; Song, X.; Yin, J.; Zhang, G.; Fei, H. Angew. Chem. Int. Ed. 2019, 58, 7818.

[2] He, J.; Zeller, M.; Hunter, A. D.; Xu, Z. J. Am. Chem. Soc. 2012, 134, 1553.

[3] Peedikakkal, A. M. P.; Quah, H. S.; Chia, S.; Jalilov, A. S.; Shaikh, A. R.; Al-Mohsin, H. A.; Yadava, K.; Ji, W.; Vittal, J. J. Inorg. Chem. 2018, 57, 11341.

[4] Lu, J.; Xin, X. H.; Lin, Y. J.; Wang, S. H.; Xu, J. G.; Zheng, F. K.; Guo, G. C. Dalton Trans. 2019, 48, 1722.

[5] Lestari, W. W.; Lönnecke, P.; Sárosi, M. B.; Streit, H. C.; Adlung, M.; Wickleder, C.; Handke, M.; Einicke, W.-D.; Gläser, R.; Hey-Hawkins, E. CrystEngComm 2013, 15, 3874.

[6] Fan, Y.; Sun, J. Y.; Zhang, D. J.; Wang, L.; Cao, Y.; Li, D.; Zhang, L.Y.; Song, W.; Xu, J.-N. CrystEngComm 2012, 14, 3982.

[7] Chen, Q.; Chen, S. C.; Zhang, Z. H.; Zhou, Y. S.; Zhou, W. Y.; Li, Y. Z.; He, M. Y.; Du, M. Cryst. Growth Des. 2011, 11, 4190.

[8] Wibowo, A. C.; Vaughn, S. A.; Smith, M. D.; Zur Loye, H. C. Inorg. Chem. 2010, 49, 11001.

[9] Cui, J.; Wong, Y. L.; Zeller, M.; Hunter, A. D.; Xu, Z. Angew. Chem. Int. Ed. 2014, 53, 14438.

[10] Bai, D. J.; He, Y. B.; Jiang, D. H. Inorg. Chem. Front. 2017, 4, 1501.

[11] Dong, Y.; Li, Y.; Wei, Y. L.; Wang, J. C.; Ma, J. P.; Ji, J.; Yao, B. J.; Dong, Y. B. Chem. Commun. 2016, 52, 10505.

[12] Yang, Q.; Yang, G.; Zhang, W.; Zhang, S.; Yang, Z.; Xie, G.; Wei, Q.; Chen, S.; Gao, S. Chem. Eur. J. 2017, 23,9149 .

[13] Dai, F.; Fan, W.; Bi, J.; Jiang, P.; Liu, D.; Zhang, X.; Lin, H.; Gong, C.; Wang, R.; Zhang, L.; Sun, D. Dalton Trans. 2016, 45, 61.

[14] Shang, Y.; Jin, B.; Peng, R.; Liu, Q.; Tan, B.; Guo, Z.; Zhao, J.; Zhang, Q. Dalton Trans. 2016, 45, 13881.

[15] Lin, X. M.; Li, T. T.; Chen, L. F.; Zhang, L.; Su, C. Y. Dalton Trans. 2012, 41, 10422. 
Table S2 Crystal data and refinement details for the compounds 1 3.

\begin{tabular}{|c|c|c|c|}
\hline Compounds & 1 & 2 & 3 \\
\hline Formula & $\mathrm{C}_{38} \mathrm{H}_{41} \mathrm{~N}_{5} \mathrm{O}_{11} \mathrm{~Pb}_{2}$ & $\mathrm{C}_{33.5} \mathrm{H}_{31.5} \mathrm{~N}_{4.5} \mathrm{O}_{10.5} \mathrm{~Pb}_{2}$ & $\mathrm{C}_{35} \mathrm{H}_{36} \mathrm{~N}_{4} \mathrm{O}_{9} \mathrm{~Pb}_{2}$ \\
\hline Temperature / K & 100 & 100 & 100 \\
\hline Formula weight & 1158.2 & 1079.5 & 1071.1 \\
\hline Radiation / $\AA$ & 0.71073 & 0.71073 & 0.71073 \\
\hline Crystal system & Triclinic & Triclinic & Triclinic \\
\hline Space group & $P \overline{1}$ & $P \overline{1}$ & $P \overline{1}$ \\
\hline$a / \AA$ & $10.3203(2)$ & $10.3027(2)$ & $10.2850(3)$ \\
\hline$b / \AA$ & $13.2585(3)$ & $13.2085(3)$ & $13.3070(3)$ \\
\hline$c / \AA$ & $13.5030(3)$ & $13.4634(3)$ & $13.3260(3)$ \\
\hline$\alpha /{ }^{\circ}$ & $93.919(2)$ & $94.2432(18)$ & $102.181(2)$ \\
\hline$\beta /{ }^{\circ}$ & $94.862(2)$ & $94.4958(16)$ & $97.698(2)$ \\
\hline$\gamma /{ }^{\circ}$ & $98.199(2)$ & $98.3760(18)$ & $94.222(2)$ \\
\hline$V / \AA^{3}$ & $1816.10(7)$ & $1800.24(7)$ & $1756.90(8)$ \\
\hline$Z$ & 2 & 2 & 2 \\
\hline$\rho_{\text {calcd }} \mathrm{g} / \mathrm{cm}^{-3}$ & 1.794 & 2.800 & 1.929 \\
\hline$\mu \mathrm{mm}^{-1}$ & 9.31 & 18.70 & 9.63 \\
\hline $\mathrm{F}(000)$ & 914 & 1384 & 962 \\
\hline Measured reflections & 25486 & 47713 & 20084 \\
\hline$R_{\text {int }}$ & 0.033 & 0.050 & 0.036 \\
\hline $\begin{array}{l}\text { Independent } \\
\text { reflections }\end{array}$ & 9057 & 9401 & 6999 \\
\hline$S$ & 1.06 & 1.11 & 1.09 \\
\hline$R_{I}^{\mathrm{a}}$ & 0.030 & 0.035 & 0.033 \\
\hline$w R_{2}^{\mathrm{b}}$ & 0.074 & 0.097 & 0.099 \\
\hline Void fraction & $29.0 \%$ & $29.4 \%$ & $27.7 \%$ \\
\hline CCDC number & 1943141 & 1943138 & 1943143 \\
\hline
\end{tabular}

${ }^{a} R_{1}=\sum|| F_{\mathrm{o}}|-| F_{\mathrm{c}}|| \sum\left|F_{\mathrm{o}}\right| \cdot{ }^{b} w R_{2}=\left[\sum w\left(F_{\mathrm{o}}^{2}-F_{\mathrm{c}}^{2}\right)^{2} / \sum w\left(F_{\mathrm{o}}{ }^{2}\right)^{2}\right]^{1 / 2}$. 
Table S3 The selected bond lengths $(\AA)$ and bond angles $\left(^{\circ}\right)$ of $\mathbf{1}$.

\begin{tabular}{|c|c|c|c|}
\hline $\mathrm{Pb} 1-\mathrm{O} 1$ & $2.440(2)$ & $\mathrm{Pb} 2-\mathrm{O} 2^{\text {viii }}$ & $2.845(2)$ \\
\hline $\mathrm{Pb} 1-\mathrm{O} 2$ & $2.643(2)$ & $\mathrm{Pb} 2-\mathrm{O}^{\mathrm{ii}}$ & $2.635(2)$ \\
\hline $\mathrm{Pb} 1-\mathrm{O} 3$ & $2.804(2)$ & $\mathrm{Pb} 2-\mathrm{O} 5$ & $2.489(2)$ \\
\hline $\mathrm{Pb} 1-\mathrm{O} 4$ & $2.449(2)$ & $\mathrm{Pb} 2-\mathrm{O} 6$ & $2.613(2)$ \\
\hline $\mathrm{Pb} 1-\mathrm{O}^{\mathrm{vi}}$ & $2.902(2)$ & $\mathrm{Pb} 2-\mathrm{O}^{\mathrm{ix}}$ & $2.797(2)$ \\
\hline $\mathrm{Pb} 1-\mathrm{O} 7^{\mathrm{vii}}$ & $2.823(3)$ & $\mathrm{Pb} 2-\mathrm{O} 7^{\mathrm{vii}}$ & $2.771(3)$ \\
\hline $\mathrm{Pb} 1-\mathrm{O} 9$ & $2.648(4)$ & $\mathrm{Pb} 2-\mathrm{O}^{\mathrm{i}}$ & $2.578(2)$ \\
\hline $\mathrm{Pb} 1-\mathrm{N} 2$ & $2.605(3)$ & $\mathrm{Pb} 2-\mathrm{N} 1^{\text {iii }}$ & $2.605(3)$ \\
\hline $\mathrm{O} 1-\mathrm{Pb} 1-\mathrm{O} 2$ & $51.68(7)$ & $\mathrm{O} 5-\mathrm{Pb} 2-\mathrm{O} 3^{\mathrm{ii}}$ & $84.21(8)$ \\
\hline $\mathrm{O} 1-\mathrm{Pb} 1-\mathrm{O} 4 \mathrm{i}$ & $82.14(8)$ & $\mathrm{O} 5-\mathrm{Pb} 2-\mathrm{O} 6$ & $51.47(8)$ \\
\hline $\mathrm{O} 1-\mathrm{Pb} 1-\mathrm{O} 9$ & $77.00(10)$ & $\mathrm{O} 5-\mathrm{Pb} 2-\mathrm{O}^{\mathrm{i}}$ & $81.14(8)$ \\
\hline $\mathrm{O} 1-\mathrm{Pb} 1-\mathrm{N} 2$ & $77.40(8)$ & $\mathrm{O} 5-\mathrm{Pb} 2-\mathrm{N} 1^{\mathrm{iii}}$ & $78.31(9)$ \\
\hline $\mathrm{O} 2-\mathrm{Pb} 1-\mathrm{O} 9$ & $99.33(10)$ & $\mathrm{O} 6-\mathrm{Pb} 2-\mathrm{O} 3^{\mathrm{ii}}$ & $99.85(7)$ \\
\hline $\mathrm{O} 4 \mathrm{i}-\mathrm{Pb} 1-\mathrm{O} 2$ & $132.99(7)$ & $\mathrm{O} 8^{\mathrm{i}}-\mathrm{Pb} 2-\mathrm{O} 3^{\mathrm{ii}}$ & $89.16(8)$ \\
\hline $\mathrm{O} 44^{\mathrm{i}-\mathrm{Pb} 1-\mathrm{O} 9}$ & $74.87(11)$ & $\mathrm{O} 8 \mathrm{i}-\mathrm{Pb} 2-\mathrm{O} 6$ & $129.87(7)$ \\
\hline $\mathrm{O} 4 \mathrm{i}-\mathrm{Pb} 1-\mathrm{N} 2$ & $76.51(9)$ & 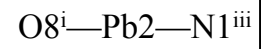 & $76.82(9)$ \\
\hline $\mathrm{N} 2-\mathrm{Pb} 1-\mathrm{O} 2$ & $84.32(8)$ & $\mathrm{N} 1{ }^{\mathrm{iii}}-\mathrm{Pb} 2-\mathrm{O} 3^{\mathrm{ii}}$ & $159.01(9)$ \\
\hline $\mathrm{N} 2-\mathrm{Pb} 1-\mathrm{O} 9$ & $143.71(10)$ & $\mathrm{N} 1{ }^{\mathrm{iii}}-\mathrm{Pb} 2-\mathrm{O} 6$ & $78.25(8)$ \\
\hline
\end{tabular}

Symmetry codes: (i) $x-1, y, z$; (ii) $-x+1,-y+1,-z$; (iii) $-x+1,-y+1,-z+1$; (iv) $x+1, y, z$;

(v) $-x+1,-y+2,-z+1$; (vi) $x+1, y+1, z$; (vii) $-x+2,-y+1,-z$; (viii) $-x+2,-y+2,-z$; (ix) $-x,-y,-z$. 
Table S4 The selected bond lengths $(\AA)$ and bond angles $\left(^{\circ}\right)$ of $\mathbf{2}$.

\begin{tabular}{|c|c|c|c|}
\hline $\mathrm{Pb} 1-\mathrm{O} 1$ & $2.452(2)$ & $\mathrm{Pb} 2-\mathrm{O} 2$ & $2.822(5)$ \\
\hline $\mathrm{Pb} 1-\mathrm{O} 2$ & $2.645(2)$ & $\mathrm{Pb} 2-\mathrm{O}^{\mathrm{ii}}$ & $2.623(2)$ \\
\hline $\mathrm{Pb} 1-\mathrm{O} 3^{\mathrm{i}}$ & $2.804(2)$ & $\mathrm{Pb} 2-\mathrm{O}^{\mathrm{vi}}$ & $2.503(2)$ \\
\hline $\mathrm{Pb} 1-\mathrm{O} 4^{\mathrm{i}}$ & $2.456(3)$ & $\mathrm{Pb} 2-\mathrm{O}^{\mathrm{ii}}$ & $2.773(5)$ \\
\hline $\mathrm{Pb} 1-\mathrm{O} 7^{\mathrm{ii}}$ & $2.821(2)$ & $\mathrm{Pb} 2-\mathrm{O}^{\mathrm{vi}}$ & $2.617(2)$ \\
\hline $\mathrm{Pb} 1-\mathrm{O} 7^{\mathrm{v}}$ & $2.863(2)$ & $\mathrm{Pb} 2-\mathrm{O}^{\mathrm{i}}$ & $2.746(2)$ \\
\hline $\mathrm{Pb} 1-\mathrm{O} 9$ & $2.611(3)$ & $\mathrm{Pb} 2-\mathrm{O}^{\mathrm{i}}$ & $2.586(3)$ \\
\hline $\mathrm{Pb} 1-\mathrm{N} 2$ & $2.549(3)$ & $\mathrm{Pb} 2-\mathrm{N} 1^{\mathrm{iv}}$ & $2.624(3)$ \\
\hline $\mathrm{O} 1-\mathrm{Pb} 1-\mathrm{O} 2$ & $51.47(8)$ & $\mathrm{O} 5-\mathrm{Pb} 2-\mathrm{O} 6$ & $51.15(8)$ \\
\hline $\mathrm{O} 1-\mathrm{Pb} 1-\mathrm{O} 4^{\mathrm{i}}$ & $81.99(8)$ & $\mathrm{O} 5-\mathrm{Pb} 2-\mathrm{O}^{\mathrm{i}}$ & $126.46(8)$ \\
\hline $\mathrm{O} 1-\mathrm{Pb} 1-\mathrm{O} 9$ & $78.78(10)$ & $\mathrm{O} 5-\mathrm{Pb} 2-\mathrm{O}^{\mathrm{i}}$ & $81.68(8)$ \\
\hline $\mathrm{O} 1-\mathrm{Pb} 1-\mathrm{N} 2$ & $77.83(9)$ & $\mathrm{O} 5-\mathrm{Pb} 2-\mathrm{N} 1^{\mathrm{iv}}$ & $78.12(9)$ \\
\hline $\mathrm{O} 4-\mathrm{Pb} 1-\mathrm{O} 2$ & $132.91(8)$ & $\mathrm{O} 6-\mathrm{Pb} 2-\mathrm{O} 3^{\mathrm{ii}}$ & $100.10(8)$ \\
\hline $\mathrm{O} 4-\mathrm{Pb} 1-\mathrm{O} 9$ & $79.14(11)$ & $\mathrm{O} 6-\mathrm{Pb} 2-\mathrm{O}^{\mathrm{i}}$ & $177.03(8)$ \\
\hline $\mathrm{O} 4-\mathrm{Pb} 1-\mathrm{N} 2$ & $77.79(10)$ & $\mathrm{O} 6-\mathrm{Pb} 2-\mathrm{N} 1^{\text {iv }}$ & $76.99(9)$ \\
\hline $\mathrm{O} 9-\mathrm{Pb} 1-\mathrm{O} 2$ & $96.13(10)$ & 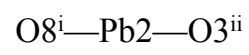 & $90.08(8)$ \\
\hline $\mathrm{N} 2-\mathrm{Pb} 1-\mathrm{O} 2$ & $84.85(8)$ & $\mathrm{O} 8 \mathrm{i}-\mathrm{Pb} 2-\mathrm{O} 6$ & $129.60(7)$ \\
\hline $\mathrm{N} 2-\mathrm{Pb} 1-\mathrm{O} 9$ & $149.06(10)$ & $\mathrm{O} 8^{\mathrm{i}}-\mathrm{Pb} 2-\mathrm{O}^{\mathrm{i}}$ & $49.06(7)$ \\
\hline $\mathrm{O} 3^{\mathrm{ii}}-\mathrm{Pb} 2-\mathrm{O} 7^{\mathrm{i}}$ & $77.52(8)$ & $\mathrm{O} 8^{\mathrm{i}}-\mathrm{Pb} 2-\mathrm{N} 1^{\mathrm{iv}}$ & $76.75(9)$ \\
\hline 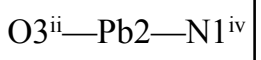 & $159.05(10)$ & $\mathrm{N} 1^{\mathrm{iv}}-\mathrm{Pb} 2-\mathrm{O}^{\mathrm{i}}$ & $104.58(9)$ \\
\hline $\mathrm{O} 5-\mathrm{Pb} 2-\mathrm{O}^{\mathrm{ii}}$ & $83.97(8)$ & & \\
\hline
\end{tabular}

Symmetry codes: (i) $x-1, y, z$; (ii) $-x-1,-y+1,-z-1$; (iii) $x+1, y, z$; (iv) $-x-1,-y+1,-z$;

(v) $x, y+1, z$; (vi) $x+1, y+1, z$. 
Table S5 The selected bond lengths $(\AA)$ and bond angles $\left(^{\circ}\right)$ of $\mathbf{3}$.

\begin{tabular}{|c|c|c|c|}
\hline $\mathrm{Pb} 1-\mathrm{O} 1$ & $2.4607(14)$ & $\mathrm{Pb} 2-\mathrm{O} 2$ & $2.8304(15)$ \\
\hline $\mathrm{Pb} 1-\mathrm{O} 2$ & $2.6251(14)$ & $\mathrm{Pb} 2-\mathrm{O} 3^{\mathrm{iii}}$ & $2.6034(14)$ \\
\hline $\mathrm{Pb} 1-\mathrm{O} 3^{\mathrm{i}}$ & $2.7762(13)$ & $\mathrm{Pb} 2-\mathrm{O} 5$ & $2.5035(14)$ \\
\hline $\mathrm{Pb} 1-\mathrm{O} 4^{\mathrm{i}}$ & $2.4719(15)$ & $\mathrm{Pb} 2-\mathrm{O} 6$ & $2.6293(13)$ \\
\hline $\mathrm{Pb} 1-\mathrm{O}^{\mathrm{v}}$ & $2.8285(14)$ & $\mathrm{Pb} 2-\mathrm{O}^{\mathrm{vi}}$ & $2.7715(13)$ \\
\hline $\mathrm{Pb} 1-\mathrm{O}^{\mathrm{vi}}$ & $2.8395(13)$ & $\mathrm{Pb} 2-\mathrm{O}^{\mathrm{i}}$ & $2.7278(13)$ \\
\hline $\mathrm{Pb} 1-\mathrm{O} 9$ & $2.6027(16)$ & $\mathrm{Pb} 2-\mathrm{O}^{\mathrm{i}}$ & $2.5513(14)$ \\
\hline $\mathrm{Pb} 1-\mathrm{N} 2$ & $2.6269(18)$ & $\mathrm{Pb} 2-\mathrm{N} 1^{\mathrm{iv}}$ & $2.6283(17)$ \\
\hline $\mathrm{O} 2-\mathrm{Pb} 1-\mathrm{N} 2$ & $84.89(5)$ & 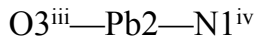 & $161.02(5)$ \\
\hline $\mathrm{O} 4 \mathrm{i}-\mathrm{Pb} 1-\mathrm{O} 2$ & $132.56(5)$ & $\mathrm{O} 8 \mathrm{i}-\mathrm{Pb} 2-\mathrm{O} 6$ & $129.12(4)$ \\
\hline $\mathrm{O} 4 \mathrm{i}-\mathrm{Pb} 1-\mathrm{N} 2$ & $76.33(6)$ & $\mathrm{O} 8^{\mathrm{i}}-\mathrm{Pb} 2-\mathrm{O}^{\mathrm{i}}$ & $49.45(4)$ \\
\hline $\mathrm{O} 4 \mathrm{i}-\mathrm{Pb} 1-\mathrm{O} 9$ & $77.56(5)$ & 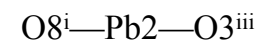 & $89.64(5)$ \\
\hline $\mathrm{O} 1-\mathrm{Pb} 1-\mathrm{O} 2$ & $51.78(5)$ & $\mathrm{O} 8^{\mathrm{i}}-\mathrm{Pb} 2-\mathrm{N} 1^{\mathrm{iv}}$ & $78.23(5)$ \\
\hline $\mathrm{O} 1-\mathrm{Pb} 1-\mathrm{O} 4^{\mathrm{i}}$ & $81.22(5)$ & $\mathrm{O} 5-\mathrm{Pb} 2-\mathrm{O} 6$ & $51.01(5)$ \\
\hline $\mathrm{O} 1-\mathrm{Pb} 1-\mathrm{N} 2$ & $75.40(5)$ & $\mathrm{O} 5-\mathrm{Pb} 2-\mathrm{O}^{\mathrm{i}}$ & $126.93(5)$ \\
\hline $\mathrm{O} 1-\mathrm{Pb} 1-\mathrm{O} 9$ & $78.29(5)$ & $\mathrm{O} 5-\mathrm{Pb} 2-\mathrm{O} 3^{\mathrm{iii}}$ & $84.58(5)$ \\
\hline $\mathrm{O} 9-\mathrm{Pb} 1-\mathrm{O} 2$ & $96.35(5)$ & $\mathrm{O} 5-\mathrm{Pb} 2-\mathrm{O}^{\mathrm{i}}$ & $81.72(5)$ \\
\hline $\mathrm{O} 9-\mathrm{Pb} 1-\mathrm{N} 2$ & $145.24(6)$ & $\mathrm{O} 5-\mathrm{Pb} 2-\mathrm{N} 1^{\mathrm{iv}}$ & $79.31(5)$ \\
\hline $\mathrm{O} 6-\mathrm{Pb} 2-\mathrm{O}^{\mathrm{i}}$ & $177.75(4)$ & $\mathrm{N} 1{ }^{\mathrm{iv}}-\mathrm{Pb} 2-\mathrm{O} 6$ & $75.75(5)$ \\
\hline $\mathrm{O} 3^{\mathrm{iii} \_\mathrm{Pb} 2-\mathrm{O} 6}$ & $101.72(4)$ & $\mathrm{N} 1^{\mathrm{iv}}-\mathrm{Pb} 2-\mathrm{O} 7^{\mathrm{i}}$ & $105.06(5)$ \\
\hline 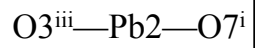 & $76.81(4)$ & & \\
\hline
\end{tabular}

Symmetry codes: (i) $x+1, y, z$; (ii) $x-1, y, z$; (iii) $-x+2,-y+3,-z+1$; (iv) $-x+2,-y+2,-z+1$; (v) $x, y, z-1$; (vi) $-x+2,-y+3,-z+1$ 
(a)

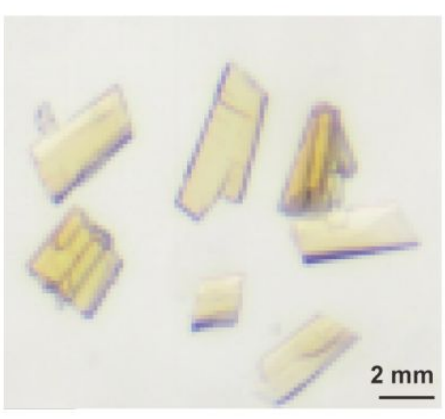

(c)

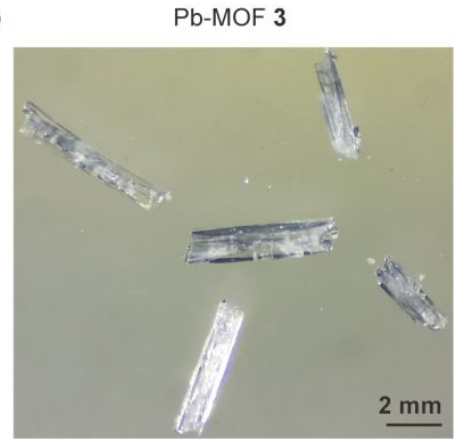

(b)

Pb-MOF 2

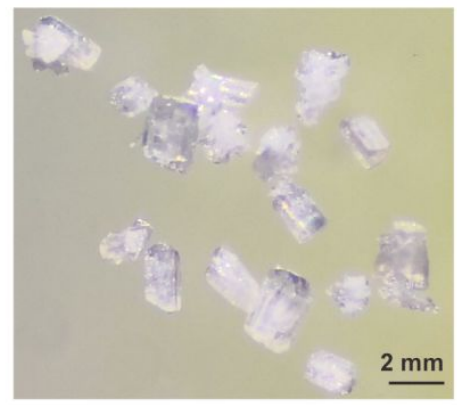

Figure S1 The photos of the as-synthesized crystals of the Pb-MOFs of $\mathbf{1}$ (a), 2 (b), and $\mathbf{3}$ (c). 
(a) Compound 1

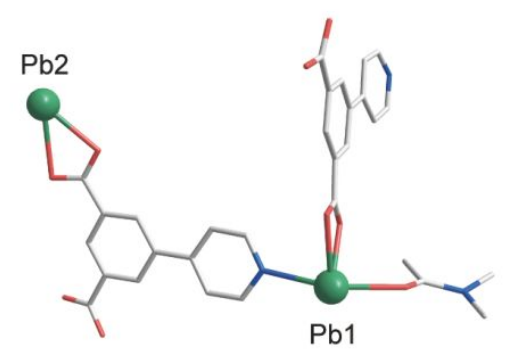

(c) Compound 3

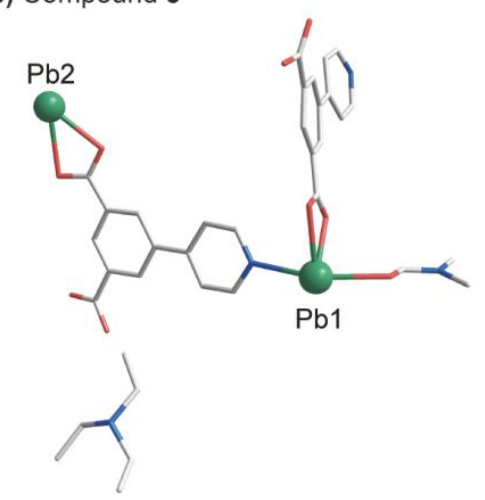

(b) Compound 2

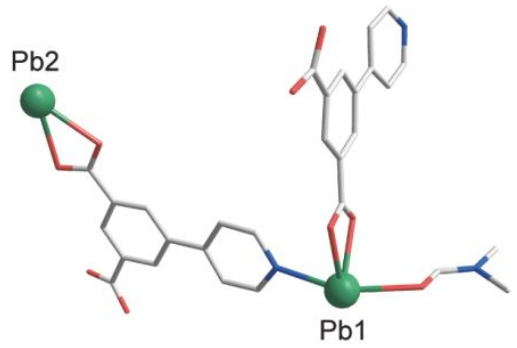

Figure S2 View of crystallographic asymmetric unit of Pb-MOFs 1 (a), 2 (b), and 3 (c). The hydrogen atoms are omitted for clarity. 


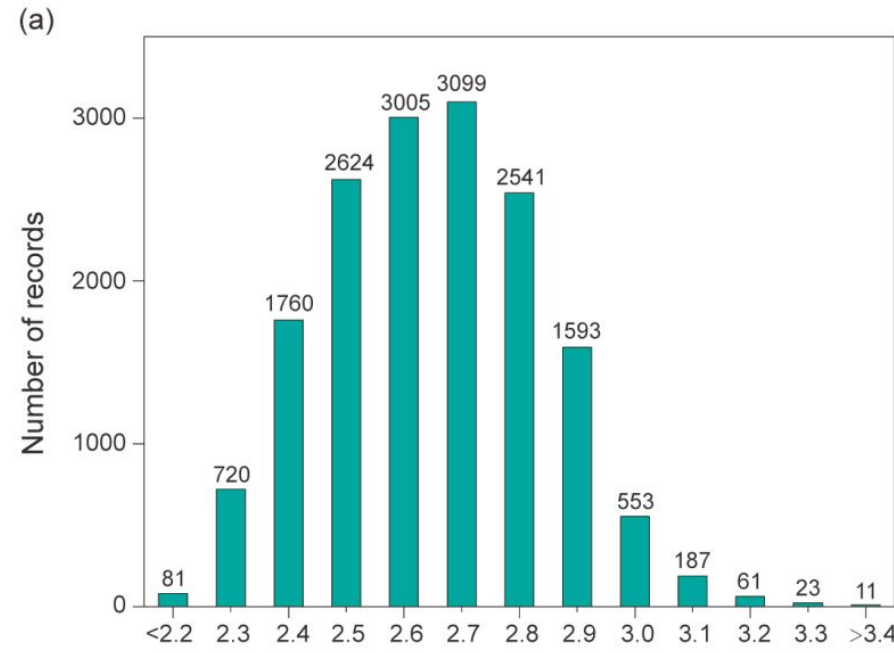

Distance of $\mathrm{Pb}-\mathrm{O}(\AA \AA)$

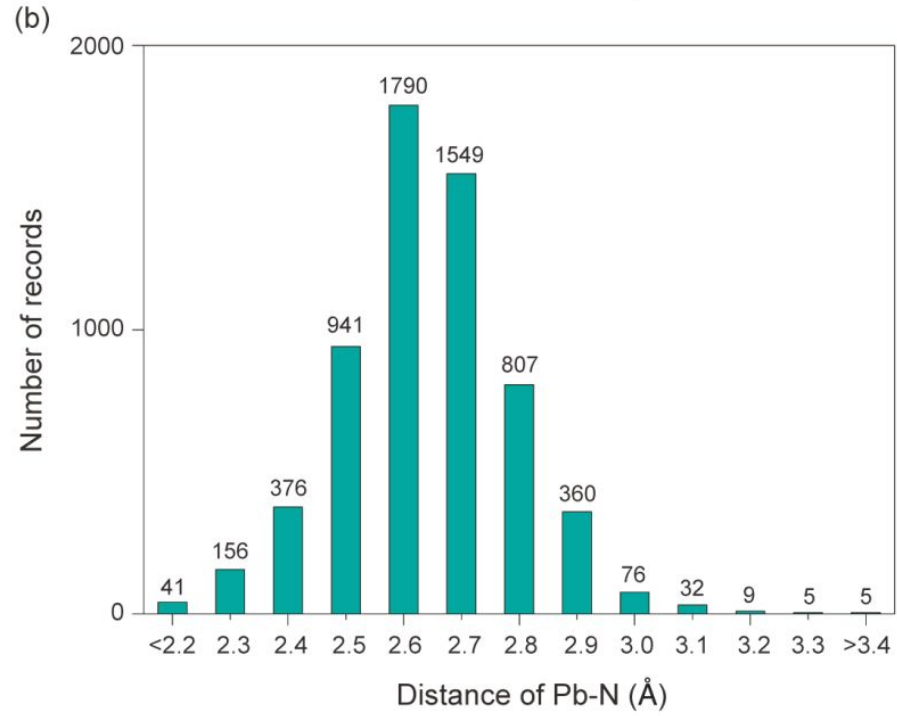

Figure S3 The statistics in histogram showing the distribution of $\mathrm{Pb}-\mathrm{O}(\mathrm{a})$ and $\mathrm{Pb}-\mathrm{N}(\mathrm{b})$ bond lengths in the CSD database (Version 2019). 
Table S6 The calculated coordination geometry of each metal centre in Pb-MOFs of $\mathbf{1} \sim \mathbf{3}$ by Shape 4.0, based on the smaller Continuous Shape Measures (CShM) value.

\begin{tabular}{|c|c|c|c|c|}
\hline Compounds & Metal ions & $\begin{array}{c}\text { Coordination } \\
\text { Numbers }\end{array}$ & Geometry & CShM Value \\
\hline \multirow{2}{*}{$\mathbf{1}$} & $\mathrm{Pb} 1$ & 8 & Biaugmented trigonal prism (BTPR-8) & 5.575 \\
\cline { 2 - 5 } & $\mathrm{Pb} 2$ & 8 & Triangular dodecahedron (TDD-8) & 4.637 \\
\hline \multirow{2}{*}{$\mathbf{2}$} & $\mathrm{Pb} 1$ & 8 & Biaugmented trigonal prism (BTPR-8) & 6.113 \\
\cline { 2 - 6 } & $\mathrm{Pb} 2$ & 8 & Triangular dodecahedron (TDD-8) & 4.479 \\
\hline \multirow{2}{*}{$\mathbf{3}$} & $\mathrm{Pb} 1$ & 8 & Biaugmented trigonal prism (BTPR-8) & 6.065 \\
\cline { 2 - 6 } & $\mathrm{Pb} 2$ & 8 & Triangular dodecahedron (TDD-8) & 4.515 \\
\hline
\end{tabular}


(a) 1D Pb-carboxylate chain

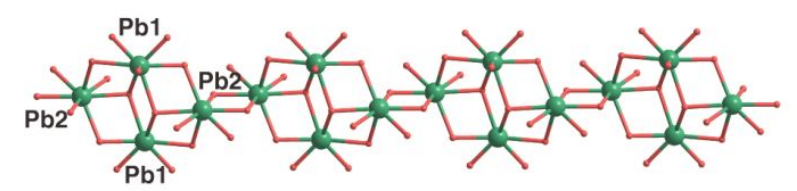

(b) $\mathrm{Pb}-\mathrm{MOF} 1$

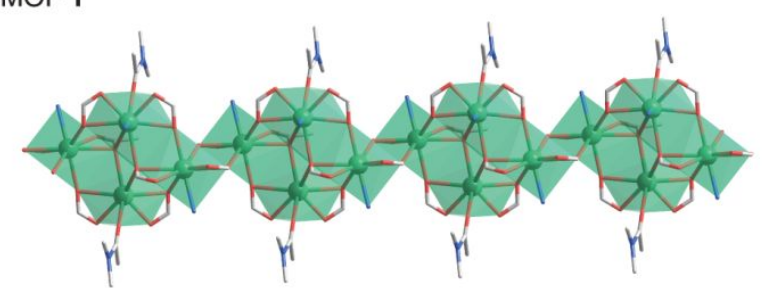

(c) $\mathrm{Pb}-\mathrm{MOF} 2$

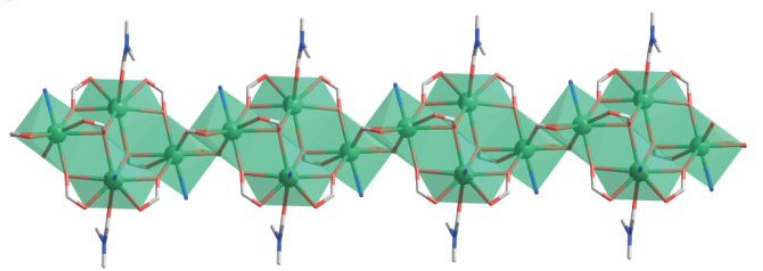

(d) Pb-MOF 3

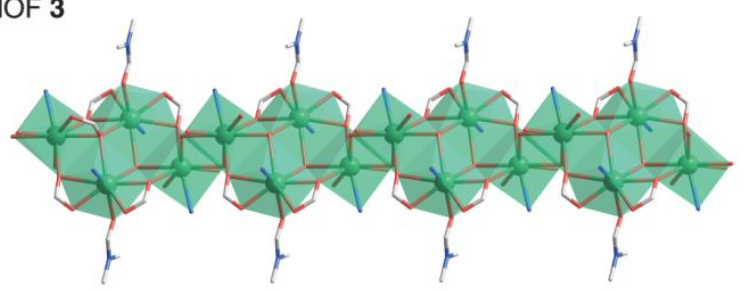

Figure S4 (a) The perspective view of the 1D Pb-carboxyl chain along $a$ axis. (b-d) The comparison of the chain structures in Pb-MOFs of $\mathbf{1} \sim \mathbf{3}$. 
(a) Pb-MOF 1

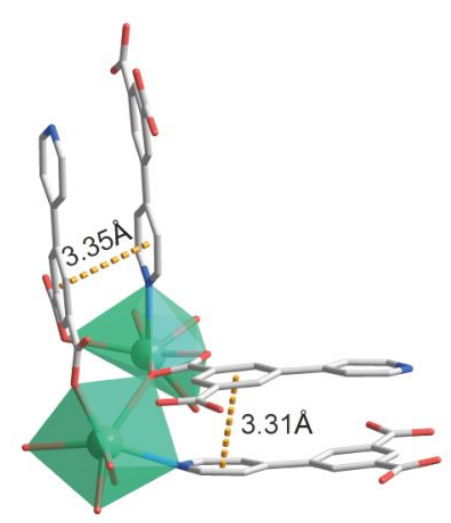

(c) Pb-MOF 3

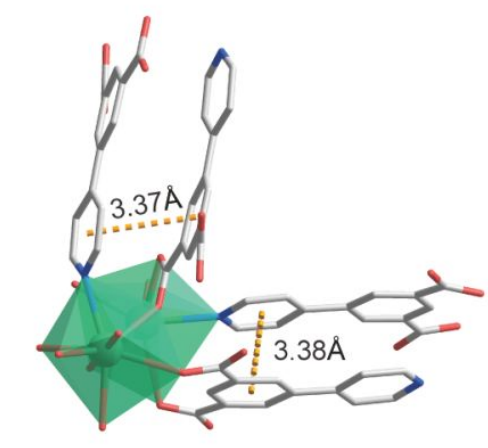

(b) Pb-MOF 2

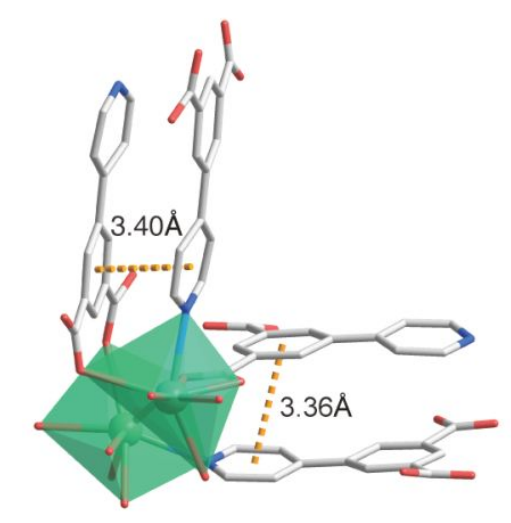

Figure S5 The perspective view of the $\pi-\pi$ interaction between the pair of pia ${ }^{2-}$ ligands in $\mathrm{Pb}-\mathrm{MOF}$ of 1 (a), 2 (b) and 3 (c). 
(a)

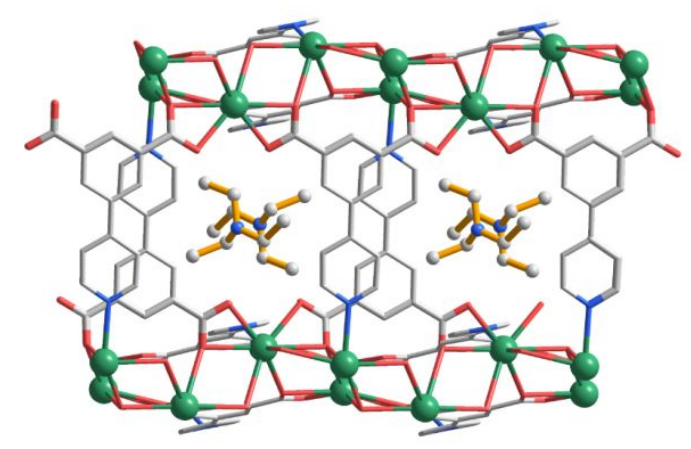

(b)

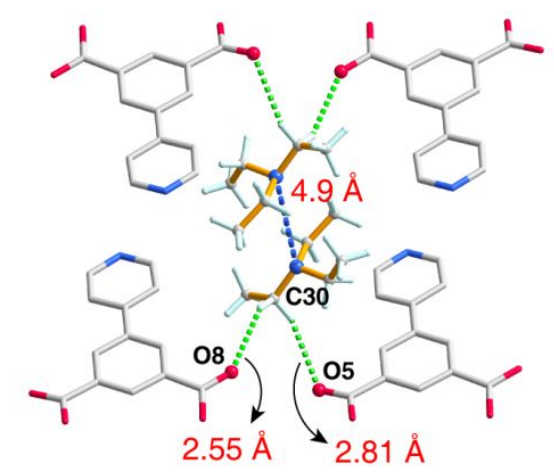

Figure S6 (a) View of the regular arrangement of $\mathrm{NEt}_{3}$ template in the 1D channel of Pb-MOF 3. (b) View of the hydrogen bonding between the $\mathrm{NEt}_{3}$ and the MOFs framework in 3 . 
(a) Pb-MOF 1

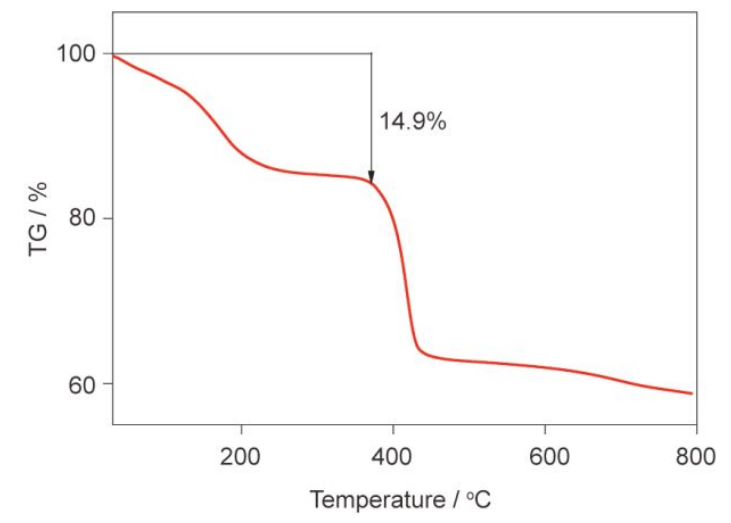

(c) Pb-MOF 3

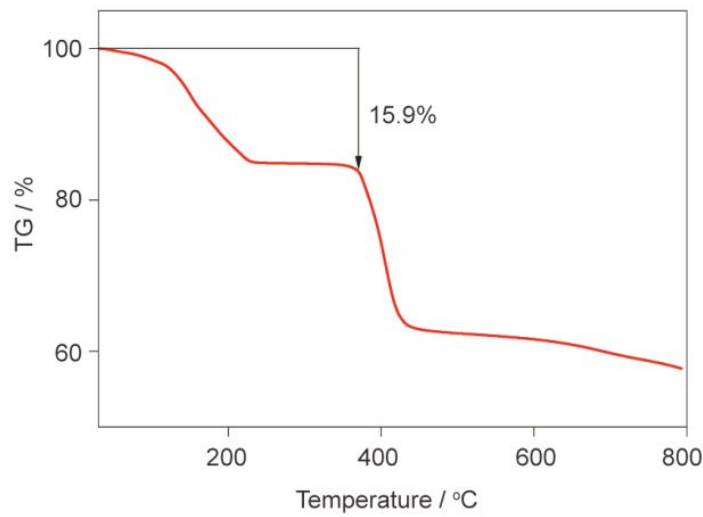

(b) $\mathrm{Pb}-\mathrm{MOF} 2$

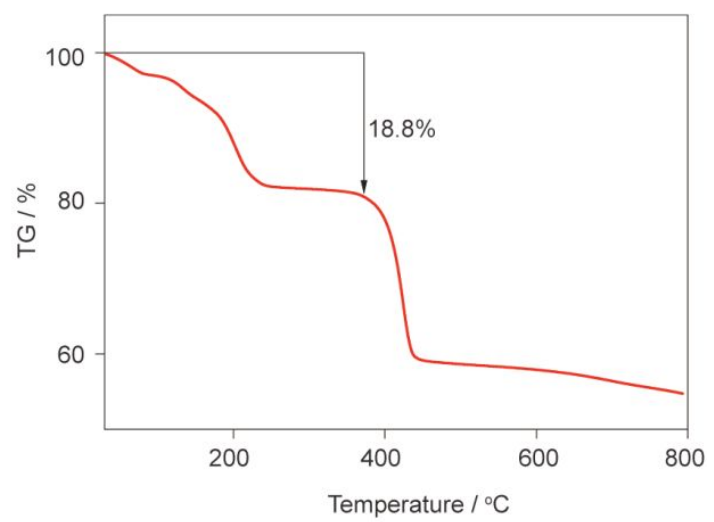

Figure $\mathbf{S} 7$ The thermogravimetric curves of Pb-MOFs of $\mathbf{1}$ (a), 2 (b), and 3 (c) under $\mathrm{N}_{2}$ atmosphere with a heating rate of $5{ }^{\circ} \mathrm{C} / \mathrm{min}$ in the temperature range of $30-800{ }^{\circ} \mathrm{C}$. As a rough analysis, the structure of 1-3 could keep almost unchanged to $370{ }^{\circ} \mathrm{C}$, the initial weight losses in the TGA curves are related to the guest and coordinated solvents. For 1, the initial weight loss of $14.9 \%$ is coincided to the calculated weight loss $(16.2 \%)$ of coordinated and guest DMA. For $\mathbf{2}$, the initial weight loss of $18.8 \%$ is coincided to the calculated weight loss $(17.0 \%)$ of coordinated and guest DMF. For $\mathbf{3}$, the initial weight loss of $15.9 \%$ is coincided to the calculated weight loss $(16.2 \%)$ of coordinated DMF and guest $\mathrm{NEt}_{3}$. 

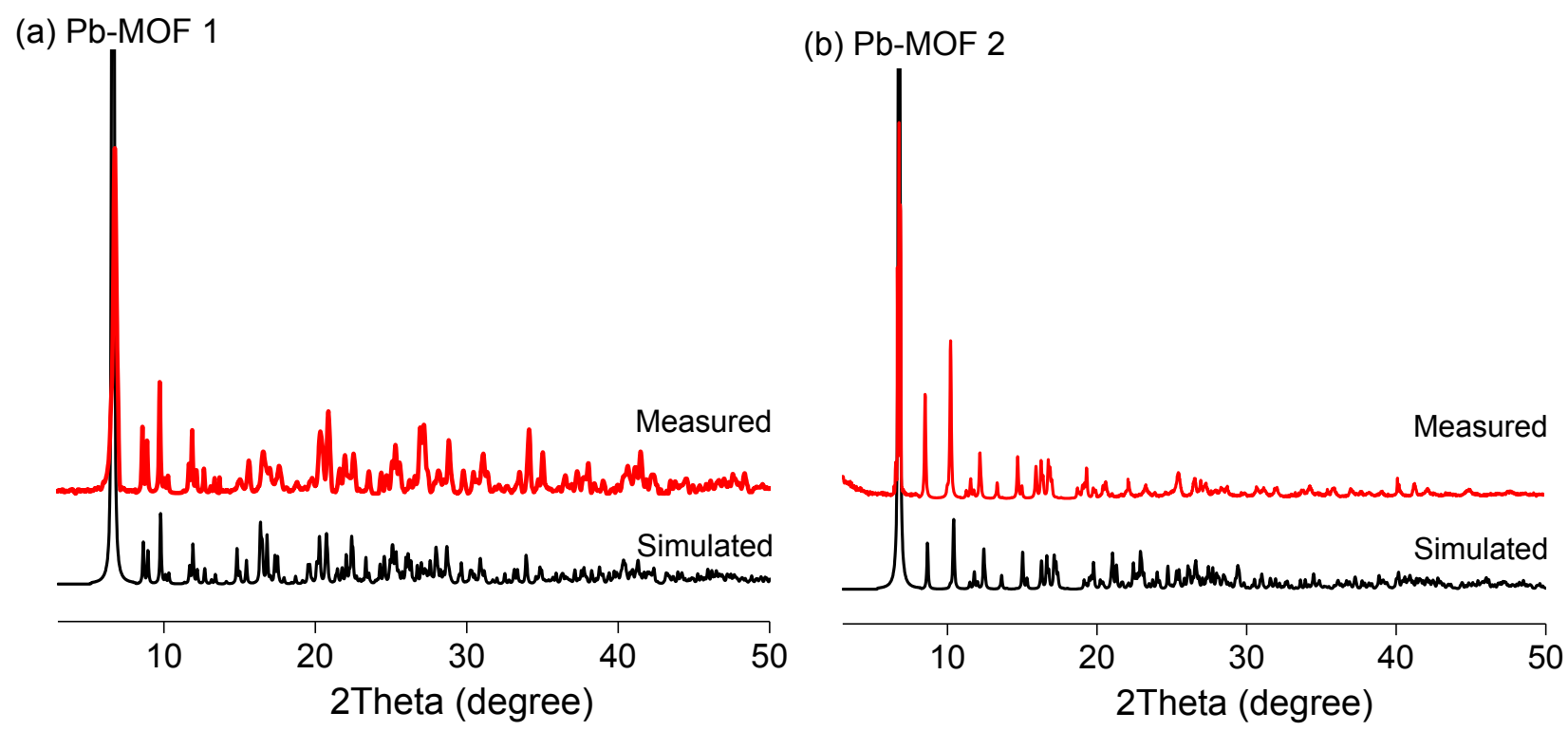

(c) $\mathrm{Pb}-\mathrm{MOF} 3$

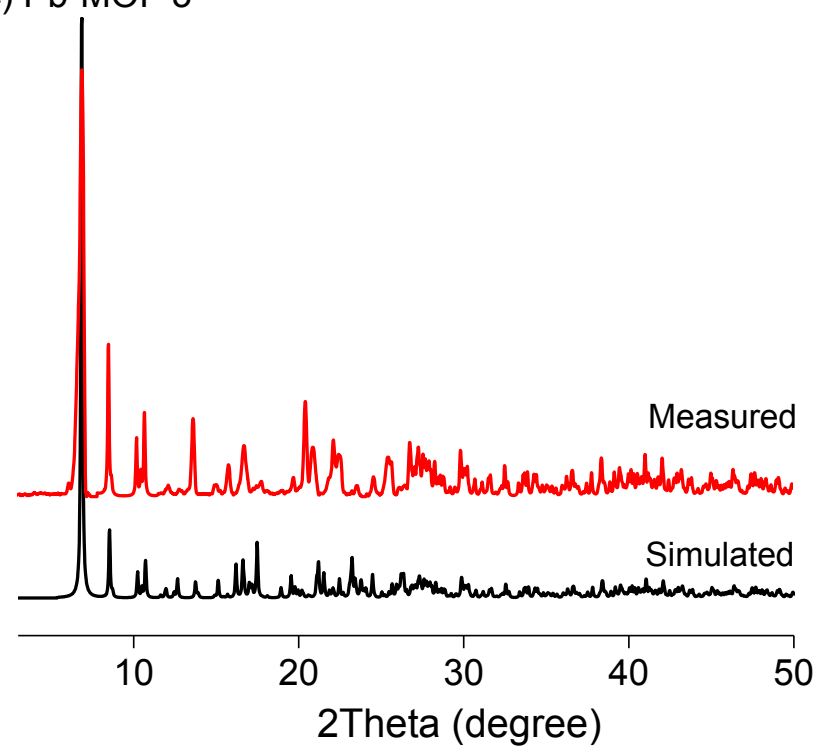

Figure S8 The simulated (black) and as-synthesized (red) PXRD patterns of the Pb-MOFs of 1 (a), 2 (b) and $\mathbf{3}$ (c). The measured results are consistent with the simulated diffraction peaks, excepting the difference in intensity of each peak due to anisotropy of the crystals. 


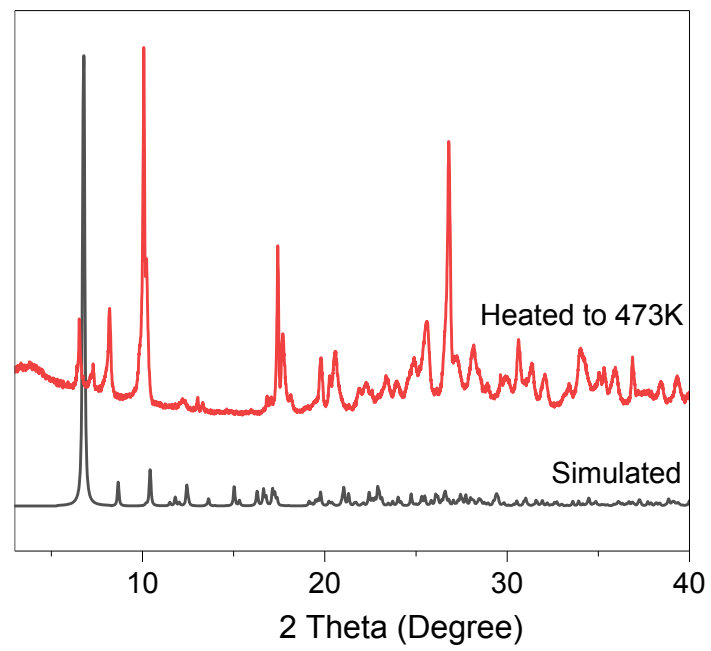

Figure S9 Comparison of the PXRD of 2 after being heated to $473 \mathrm{~K}$ to remove the guest and coordinated solvents, with the simulated patterns from the crystal structures of as-synthesized MOFs. 

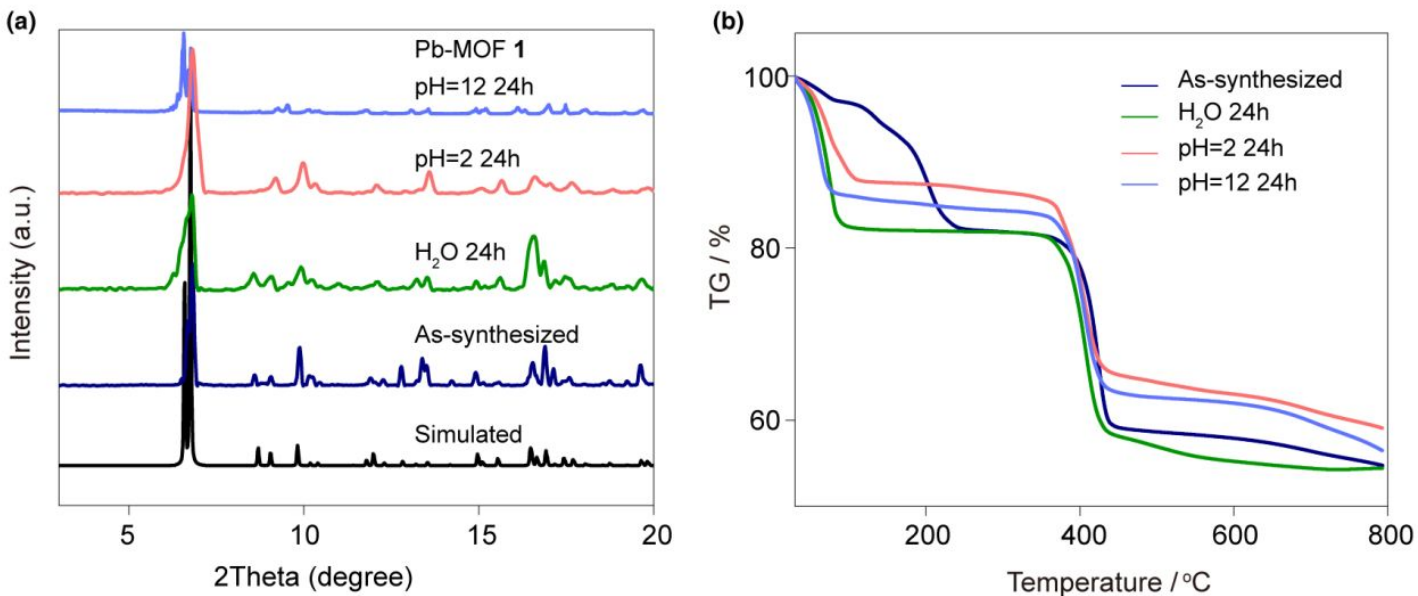

Figure S10 (a) The PXRD of Pb-MOF 1 which has been chemically treated by $\mathrm{H}_{2} \mathrm{O}, \mathrm{HCl}$ aqueous solution, and $\mathrm{NaOH}$ aqueous solution for $24 \mathrm{~h}$. The resultant measured PXRD patterns of 1 are very consistent to the simulated ones, demonstrating the framework stability. (b) TG curves of $\mathbf{1}$ after immersed in $\mathrm{H}_{2} \mathrm{O}, \mathrm{HCl}$ and $\mathrm{NaOH}$ aqueous solutions for $24 \mathrm{~h}$. 

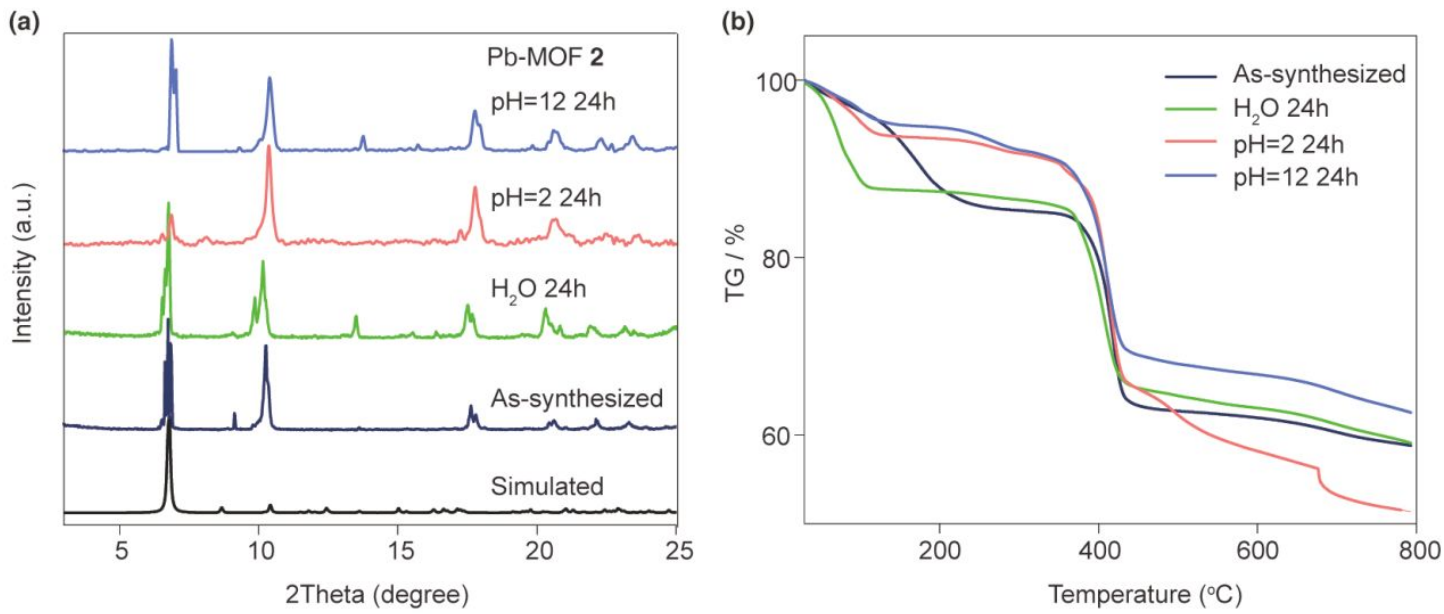

Figure S11 (a) The PXRD of Pb-MOF 2 which has been chemically treated by $\mathrm{H}_{2} \mathrm{O}, \mathrm{HCl}$ aqueous solution, and $\mathrm{NaOH}$ aqueous solution for $24 \mathrm{~h}$. The resultant measured PXRD patterns of 2 are very consistent to the simulated ones, demonstrating the framework stability. (b) TG curves of 2 after immersed in $\mathrm{H}_{2} \mathrm{O}, \mathrm{HCl}$ and $\mathrm{NaOH}$ aqueous solutions for $24 \mathrm{~h}$. 

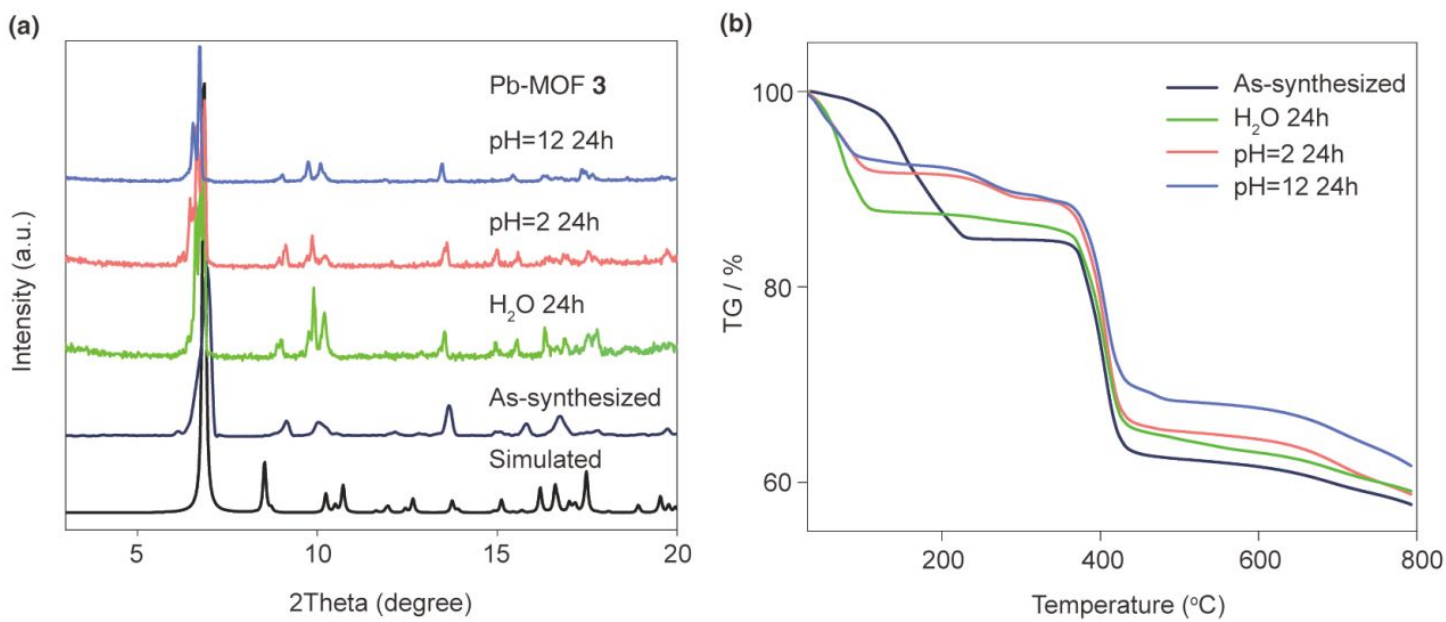

Figure $\mathrm{S12}$ (a) PXRD of $\mathrm{Pb}-\mathrm{MOF} 3$ which has been chemically treated by $\mathrm{H}_{2} \mathrm{O}, \mathrm{HCl}$ aqueous solution, and $\mathrm{NaOH}$ aqueous solution for $24 \mathrm{~h}$. The resultant measured PXRD patterns of $\mathbf{3}$ are very consistent to the simulated ones, demonstrating the framework stability. (b) TG curves of $\mathbf{3}$ after immersed in $\mathrm{H}_{2} \mathrm{O}, \mathrm{HCl}$ and $\mathrm{NaOH}$ aqueous solutions for $24 \mathrm{~h}$. 


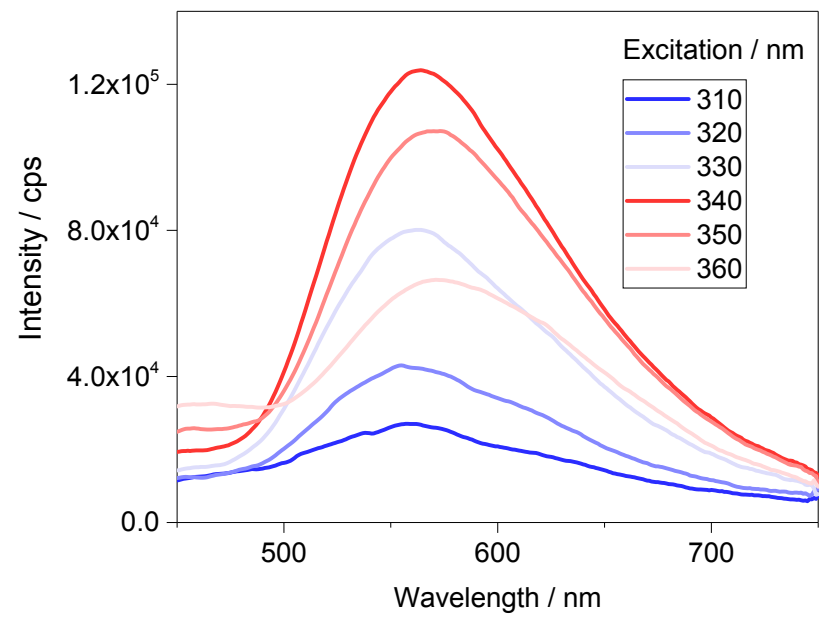

Figure S13 The solid state emission spectrum of Pb-MOF 2 under different excitations. 


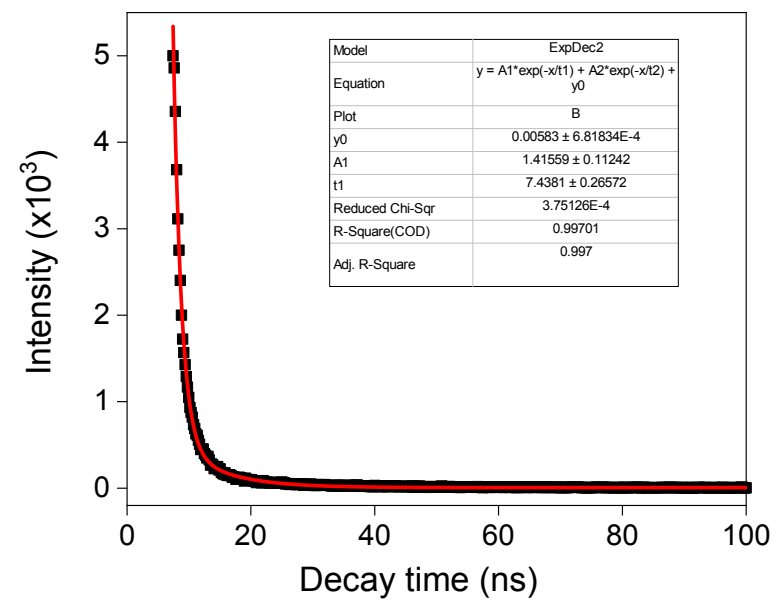

Figure S14 The decay time dependent emission intensity (black square) of the free ligand of $\mathrm{H}_{2}$ pia, giving the fitted (red line) fluorescence lifetime of $7.4 \mathrm{~ns}$. 
(a) Pb-MOF 1

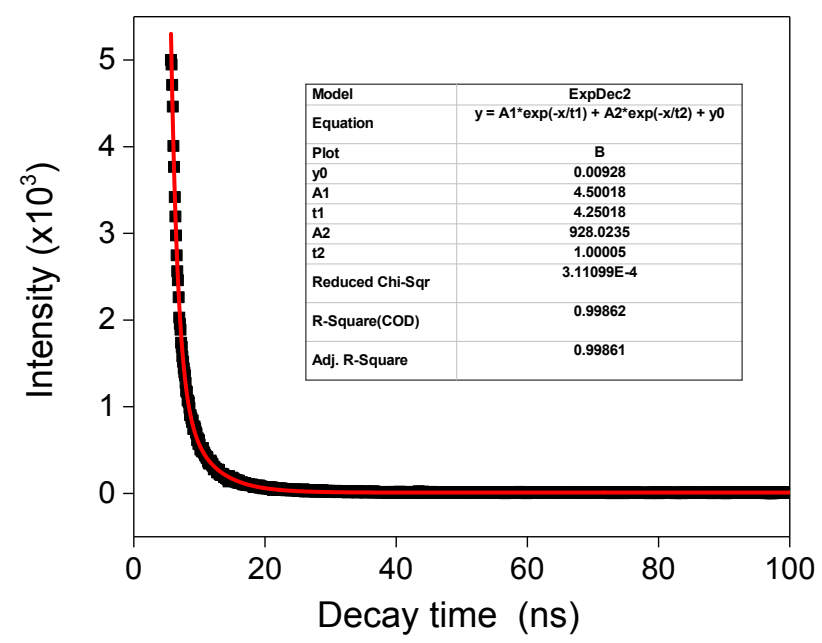

(c) Pb-MOF 3

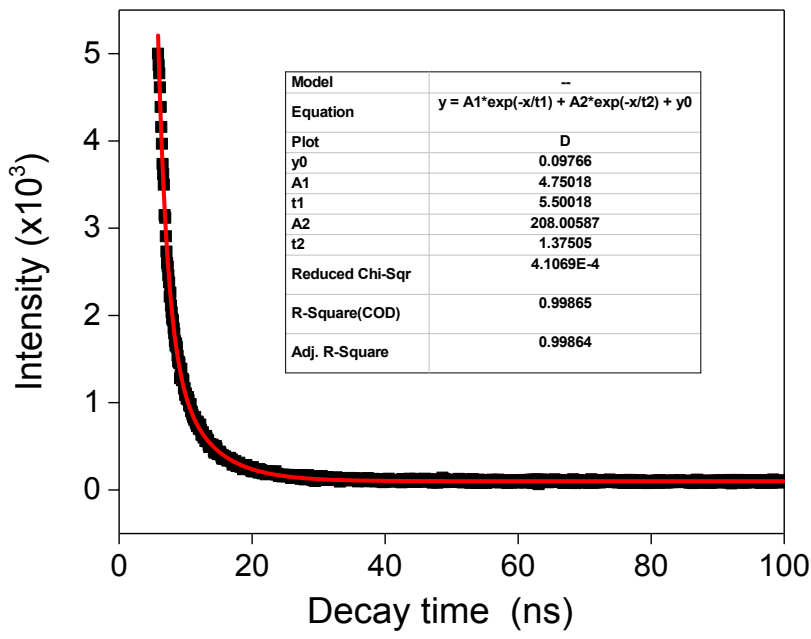

(b) $\mathrm{Pb}-\mathrm{MOF} 2$

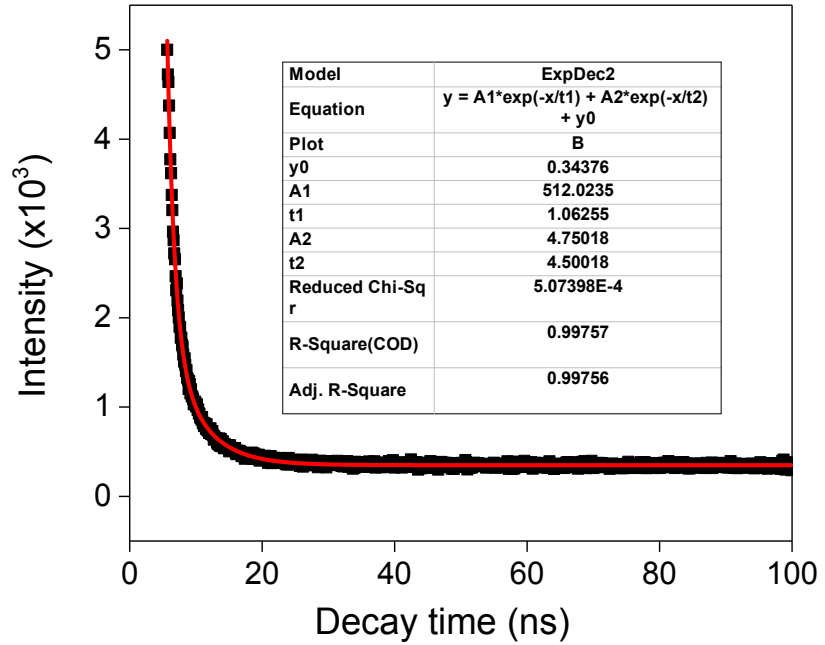

Figure S15 The decay time dependent emission intensity (black square) of the Pb-MOFs of 1 (a), 2 (b), and $\mathbf{3}$ (c). The fitted (red line) fluorescence lifetimes are 4.3, 1.1 and $5.5 \mathrm{~ns}$ for 1, 2, and 3, respectively. 

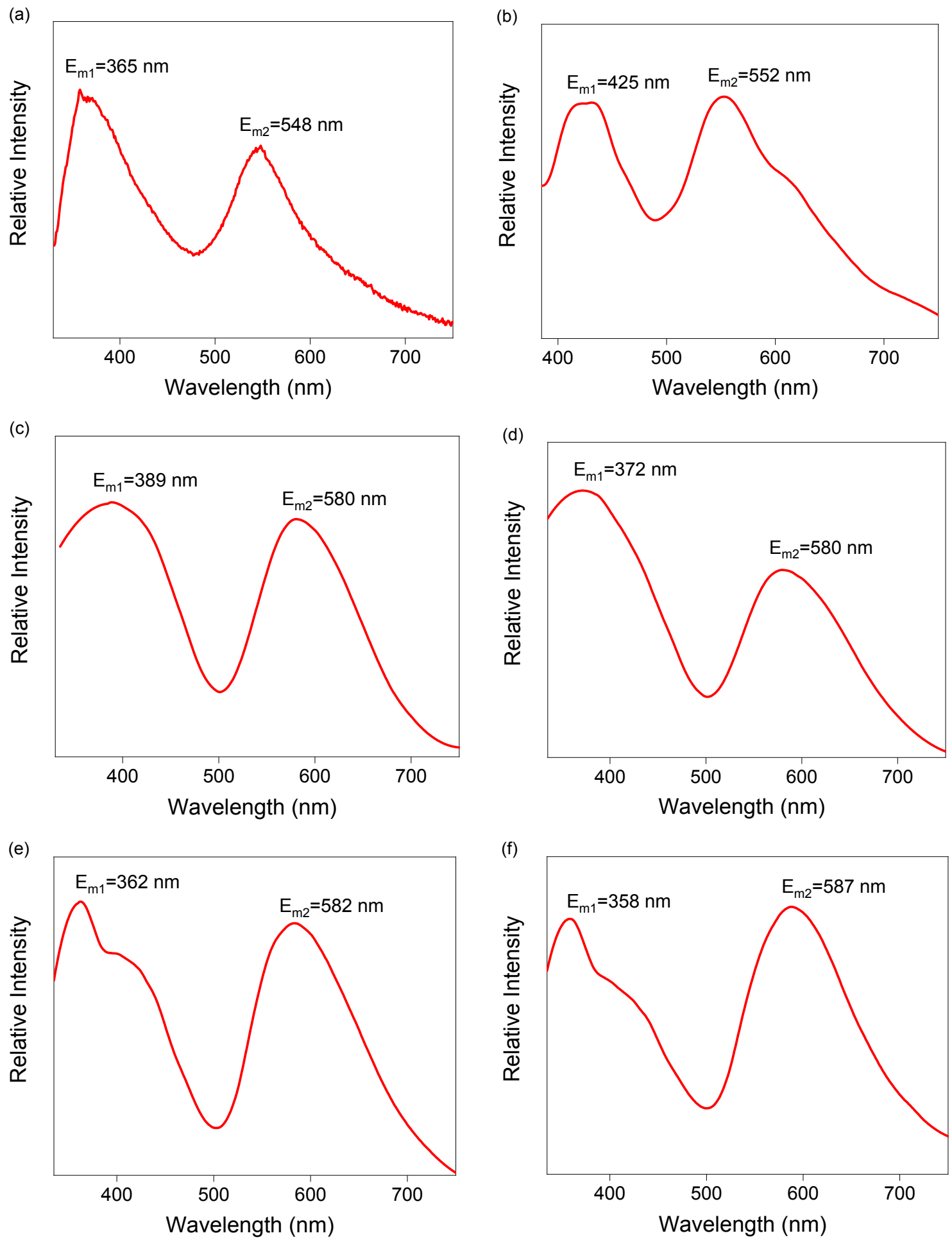

Figure S16 The solution-state emission spectra of mixture of $\mathrm{Pb}-\mathrm{MOF} 2$ and $\mathrm{H}_{2}$ pia in various solvents of (a) DMF, (b) DMA, (c) $\mathrm{CH}_{3} \mathrm{OH}$, (d) $\mathrm{C}_{2} \mathrm{H}_{5} \mathrm{OH}$, (e) $\mathrm{CH}_{2} \mathrm{Cl}_{2}$, and (f) $\mathrm{CH}_{3} \mathrm{COCH}_{3}$ under $350 \mathrm{~nm}$ excitation, which exhibited dual emissions. The suspensions were prepared by dispersing $5 \mathrm{mg}$ fineground crystals of 2 and $5 \mathrm{mg} \mathrm{H}_{2}$ pia ligand into $3 \mathrm{~mL}$ solvent, followed by ultrasonic treatment for 10 minutes. 
Table S7 Statistics of the fluorescence intensity ratio for the solution-state emission spectra of 1:1 mixture of $\mathrm{Pb}-\mathrm{MOF} 2$ and $\mathrm{H}_{2} \mathrm{Pia}$ under different solvents. $\mathrm{E}_{\mathrm{m} 1}$ and $\mathrm{E}_{\mathrm{m} 2}$ represent the emission peak of $\mathrm{H}_{2} \mathrm{Pia}$ and 2, respectively. $\mathrm{I}_{1}$ and $\mathrm{I}_{2}$ represent the relative intensity of $\mathrm{E}_{\mathrm{m} 1}$ and $\mathrm{E}_{\mathrm{m} 2}$.

\begin{tabular}{|c|c|c|c|c|}
\hline Solvent & $\mathbf{E}_{\mathbf{m} 1} / \mathbf{n m}$ & $\mathbf{E}_{\mathbf{m} \mathbf{2}} / \mathbf{n m}$ & $\mathbf{I}_{\mathbf{1}}: \mathbf{I}_{\mathbf{2}}$ & $\mathbf{C I E}$ \\
\hline $\mathrm{DMF}$ & 365 & 548 & $1: 0.88$ & $(0.315,0.349)$ \\
\hline $\mathrm{DMA}$ & 425 & 552 & $1: 1.02$ & $(0.322,0.341)$ \\
\hline $\mathrm{CH}_{3} \mathrm{OH}$ & 389 & 580 & $1: 0.94$ & $(0.364,0.327)$ \\
\hline $\mathrm{C}_{2} \mathrm{H}_{5} \mathrm{OH}$ & 372 & 580 & $1: 0.87$ & $(0.355,0.319)$ \\
\hline $\mathrm{CH}_{2} \mathrm{Cl}_{2}$ & 362 & 582 & $1: 0.93$ & $(0.379,0.340)$ \\
\hline $\mathrm{CH}_{3} \mathrm{COCH}_{3}$ & 358 & 587 & $1: 1.04$ & $(0.401,0.359)$ \\
\hline
\end{tabular}



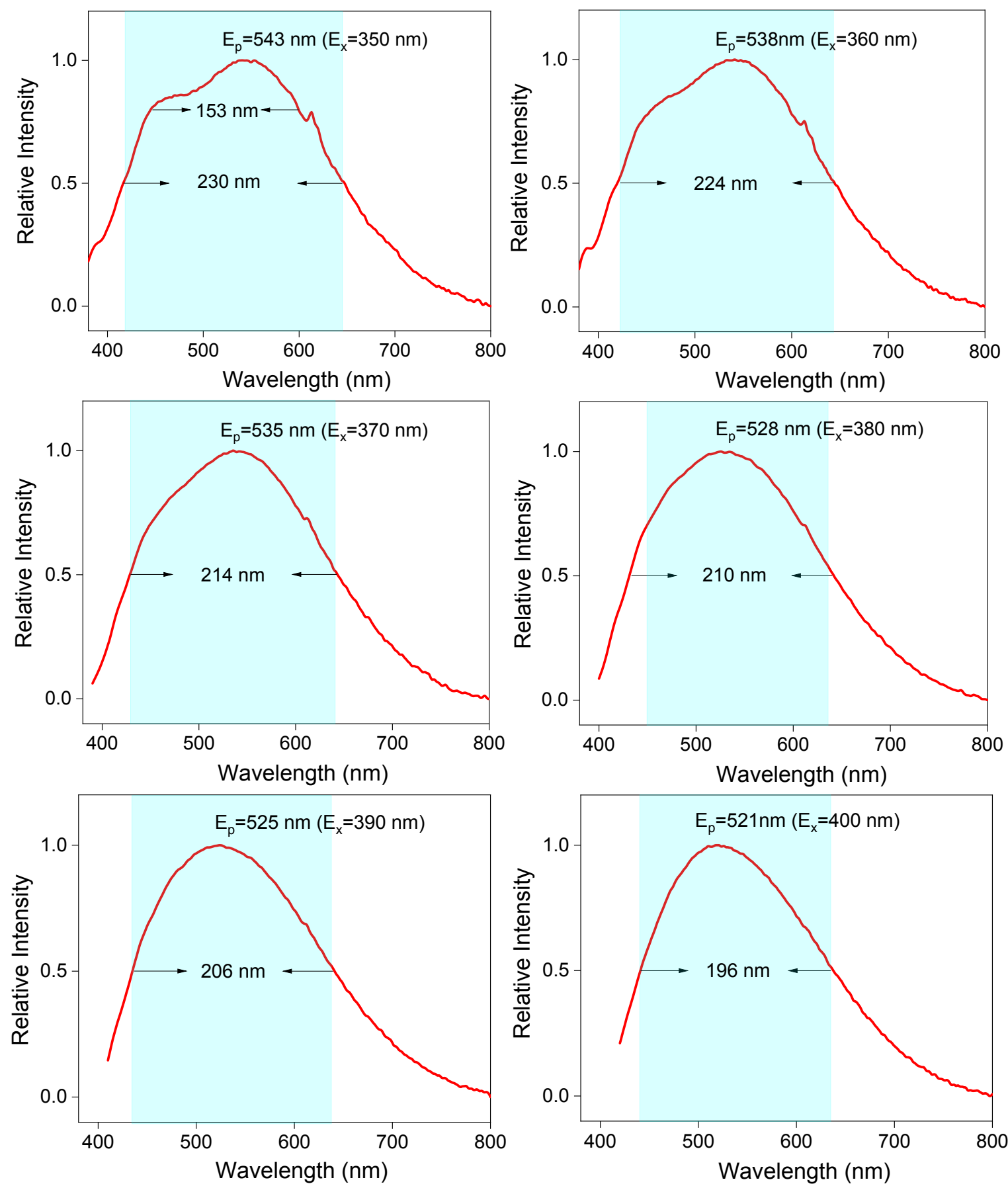

Figure S17 The solid-state fluorescence emission spectrum with the peak position $\left(\mathrm{E}_{\mathrm{p}}\right)$ of 1 under different excitation $\left(E_{x}\right)$. The region of full width at half maxima of each spectra are highlighted in cyan. 
Table S8 Statistics of the full width at half maxima (FWHM) for the solid-state emission spectra of the $\mathrm{Pb}-\mathrm{MOF} 1$ under different excitation of $350-400 \mathrm{~nm}$.

\begin{tabular}{|c|c|c|c|c|c|}
\hline $\mathbf{E}_{\mathbf{x}} / \mathbf{n m}$ & $\mathbf{E}_{\mathbf{p}} \mathbf{a} / \mathbf{n m}$ & $\mathbf{E}_{\mathbf{m} \mathbf{1}}^{\mathbf{b}} / \mathbf{n m}$ & $\mathbf{E}_{\mathbf{m} \mathbf{2}} \mathbf{c} / \mathbf{n m}$ & $\mathbf{F W H M} / \mathbf{n m}$ & $\mathbf{C I E}$ \\
\hline 350 & 543 & 417 & 647 & 230 & $(0.332,0.347)$ \\
\hline 360 & 538 & 420 & 644 & 224 & $(0.322,0.361)$ \\
\hline 370 & 535 & 429 & 643 & 214 & $(0.325,0.366)$ \\
\hline 380 & 528 & 431 & 641 & 210 & $(0.322,0.377)$ \\
\hline 390 & 525 & 435 & 641 & 206 & $(0.321,0.382)$ \\
\hline 400 & 521 & 441 & 637 & 196 & $(0.324,0.392)$ \\
\hline
\end{tabular}

${ }^{a}$ Location of the emission spectrum with the maximum intensity. ${ }^{b}$ The start point of emission spectrum with half maxima intensity. ${ }^{\mathrm{c}}$ The end point of emission spectrum with half maxima intensity. 


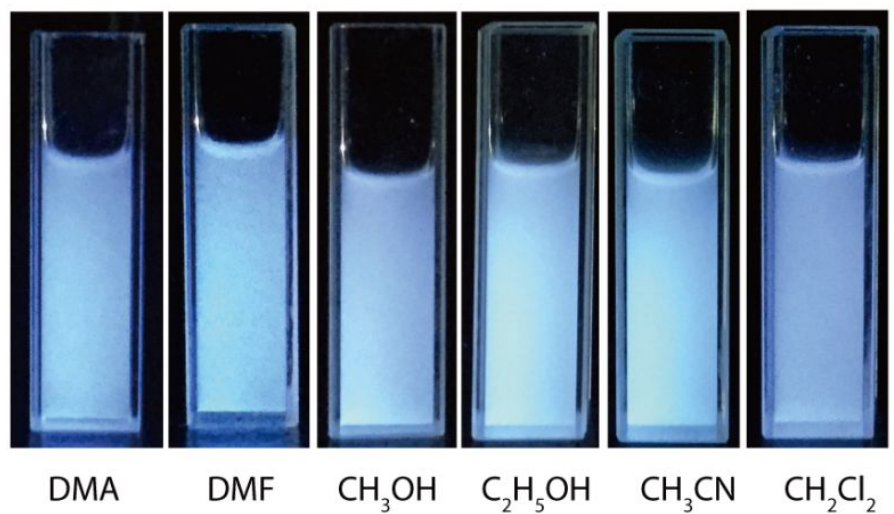

Figure S18 The photographs of the solution fluorescence of Pb-MOF 1 in different organic solvents of DMA, DMF, $\mathrm{CH}_{3} \mathrm{OH}, \mathrm{C}_{2} \mathrm{H}_{5} \mathrm{OH}, \mathrm{CH}_{3} \mathrm{CN}$ and $\mathrm{CH}_{2} \mathrm{Cl}_{2}$, under excitation of $365 \mathrm{~nm}$ using an ultraviolet lamp. The suspensions were prepared by dispersing $10 \mathrm{mg}$ fine-ground crystals of assynthesized 1 into $3 \mathrm{~mL}$ solvent, followed by ultrasonic treatment for 10 minutes. 

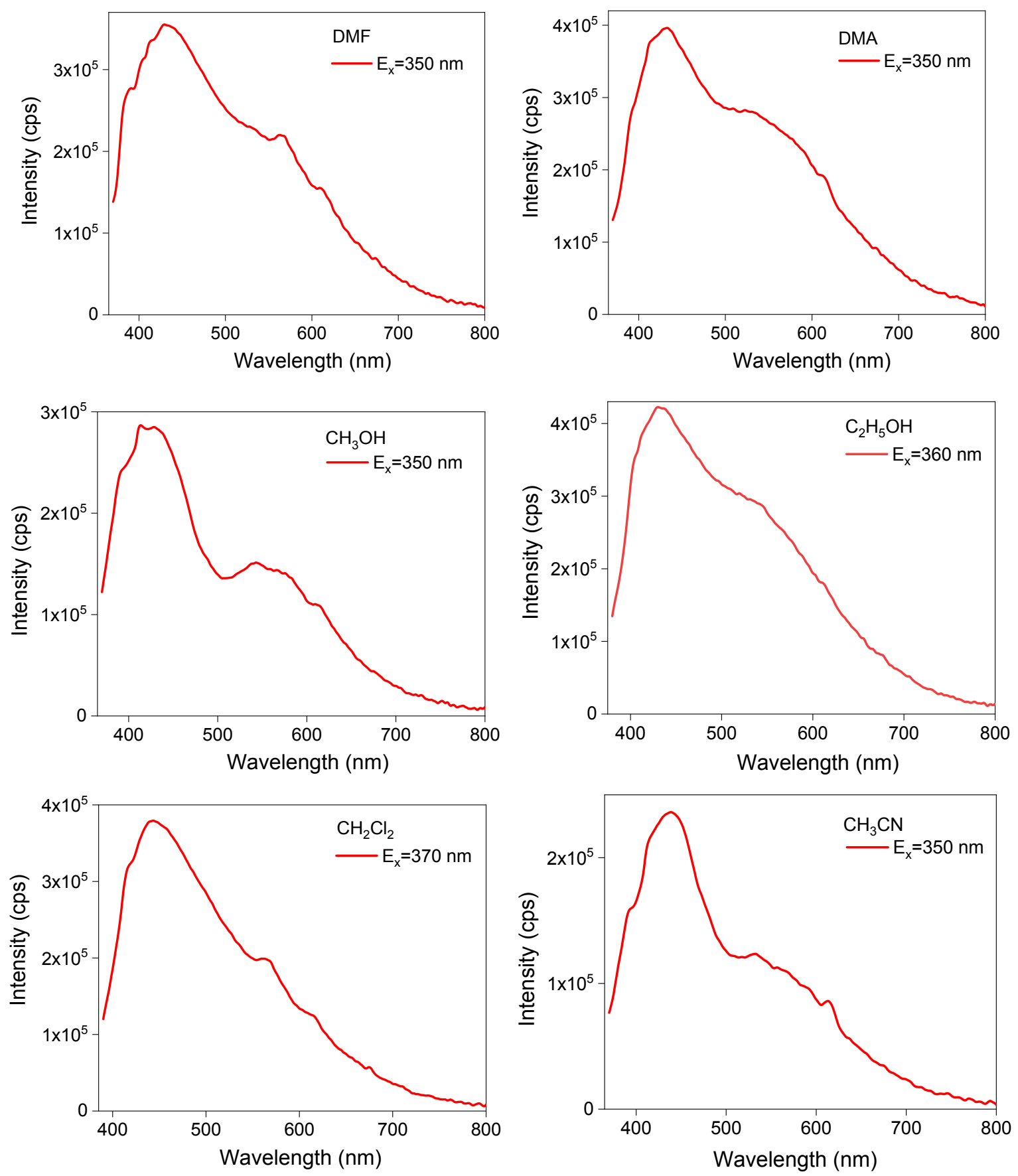

Figure S19 The solution-state emission spectra of 1 in various solvents under different excitation, which exhibit continuous and broadband spectra. The suspensions were prepared by dispersing 10 mg fine-ground crystals into $3 \mathrm{~mL}$ solvent, followed by ultrasonic treatment for 10 minutes. 


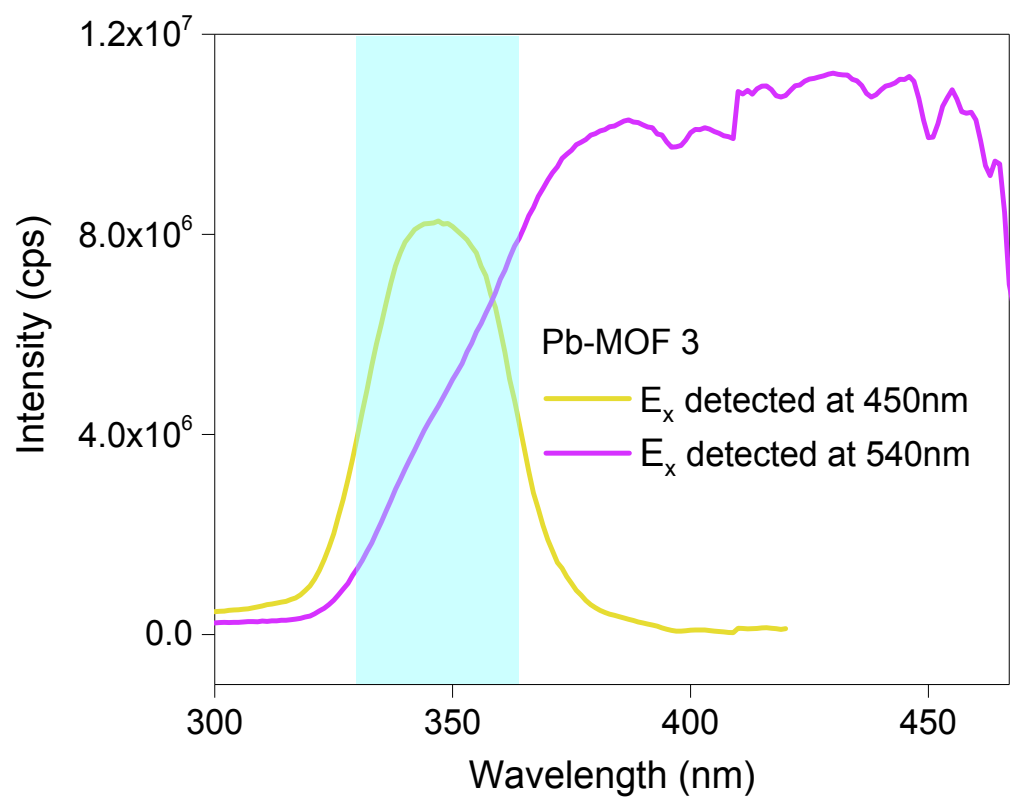

Figure S20 The solid-state fluorescence excitation spectrum of 3 detected at emission of $450 \mathrm{~nm}$ and $540 \mathrm{~nm}$. 
Table S9 Statistics of the fluorescence intensity ratio for the solid-state emission spectra of $\mathbf{3}$ under different excitation from 330 to $350 \mathrm{~nm}$.

\begin{tabular}{|c|c|c|}
\hline $\mathbf{E}_{\mathbf{x}} / \mathbf{n m}$ & Intensity $\mathbf{~ o f ~} \mathbf{E}^{\mathbf{a}} / \mathbf{E}^{\mathbf{b}}$ ratio & $\mathbf{C I E}$ \\
\hline 330 & 1.40 & $(0.302,0.394)$ \\
\hline 335 & 1.66 & $(0.290,0.283)$ \\
\hline 340 & 1.94 & $(0.275,0.272)$ \\
\hline 345 & 2.01 & $(0.266,0.266)$ \\
\hline 350 & 2.03 & $(0.259,0.263)$ \\
\hline
\end{tabular}

a The former $450 \mathrm{~nm}$ peak position of emission spectrum. ${ }^{\mathrm{b}}$ The latter $545 \mathrm{~nm}$ peak position of emission spectrum. 


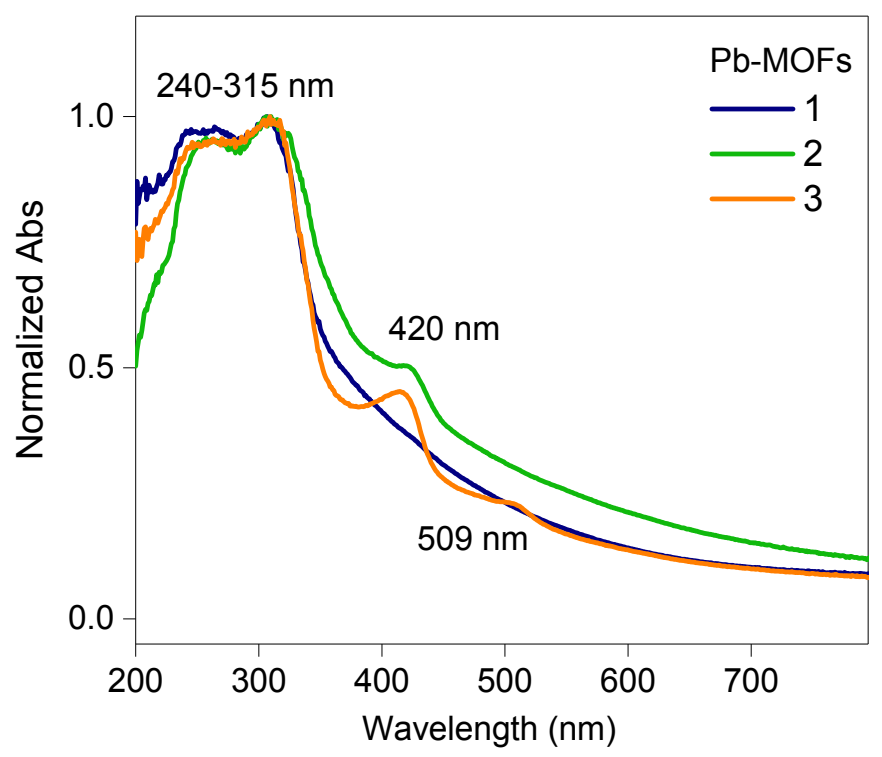

Figure S21 The solid-state UV diffuse reflectance spectroscopy of Pb-MOFs $\mathbf{1} \sim \mathbf{3}$. The high-energy and strong adsorption band within 240-315 $\mathrm{nm}$ was ascribed to ligand adsorption. The additional adsorption at $420 \mathrm{~nm}$ stemming from charge-transfer between metal and ligand, was found for $\mathbf{2}$ and 3, but not for 1. Different from 2, there was another weak at $509 \mathrm{~nm}$ for $\mathbf{3}$. These results indicated the intrinsic difference of electronic energy levels of the three MOFs. 